\title{
Puzzling wind properties of young massive stars in SMC-N81 ${ }^{\star}$
}

\author{
F. Martins ${ }^{1,2}$, D. Schaerer ${ }^{2,1}$, D. J. Hillier ${ }^{3}$, and M. Heydari-Malayeri ${ }^{4}$ \\ ${ }^{1}$ Laboratoire d'Astrophysique, Observatoire Midi-Pyrénées, 14 Av. E. Belin, 31400 Toulouse, France \\ 2 Observatoire de Genève, 51 Chemin des Maillettes, 1290 Sauverny, Switzerland \\ 3 Department of Physics and Astronomy, University of Pittsburgh, 3941 O'Hara Street, Pittsburgh, PA 15260, USA \\ ${ }^{4}$ LERMA, Observatoire de Paris, 61 Avenue de l'Observatoire, 75014 Paris, France
}

Recieved 14 October 2003 / Accepted 16 March 2004

\begin{abstract}
We present a quantitative study of massive stars in the High Excitation Blob N81, a compact star forming region in the SMC. The stellar content was resolved by HST, and STIS was used to obtain medium resolution spectra. The qualitative analysis of the stellar properties presented in Heydari-Malayeri et al. (2002a) is extended using non-LTE spherically extended atmosphere models including line-blanketing computed with the code CMFGEN (Hillier \& Miller 1998), and the wind properties are investigated. The main results are the following:

- The SMC-N81 components are young ( 0-4 Myrs) O stars with effective temperatures compatible with medium to late subtypes and with luminosities lower than those of average Galactic $\mathrm{O}$ dwarfs, rendering them possible ZAMS candidates.

- The winds are extremely weak: with values of the order of $10^{-8} / 10^{-9} M_{\odot} \mathrm{yr}^{-1}$ the mass loss rates are lower than observed so far for Galactic dwarfs. Only the recent study of SMC stars by Bouret et al. (2003) show the same trend. The modified wind momenta $\left(\dot{M} v_{\infty} \sqrt{R}\right)$ are also 1 to 2 orders of magnitude lower than observed for Galactic stars. Both the mass loss rates and the modified wind momenta are lower than the predictions of the most recent hydrodynamical models.
\end{abstract}

The accuracy of the UV based mass loss rate determination, relying in particular on the predicted ionisation fractions, are carefully examined. We find that $\dot{M}$ could be underestimated by a factor of up to 10 . Even in this unlikely case, the above conclusions remain valid qualitatively.

The reasons for such weak winds are investigated with special emphasis on the modified wind momenta:

- There may be a break-down of the wind momentum-luminosity relation (WLR) for dwarf stars at low luminosity (log $L / L_{\odot} \lesssim$ 5.5). However, reasons for such a breakdown remain unknown.

- The slope of the WLR may be steeper at low metallicity. This is predicted by the radiation driven wind theory, but the current hydrodynamical simulations do not show any change of the slope at SMC metallicity. Moreover, there are indications that some Galactic objects have wind momenta similar to those of the SMC stars.

- Decoupling may take place in the atmosphere of the SMC-N81 stars, leading to multicomponent winds. However, various tests indicate that this is not likely to be the case.

The origin of the weakness of the wind observed in the SMC-N81 stars remains unknown. We suggest that this weakness may be linked with the youth of these stars and represents possibly the onset of stellar winds in recently formed massive stars.

Key words. stars: winds, outflows - stars: atmospheres - stars: mass-loss - stars: early-type - stars: fundamental parameters ISM: HII region

\section{Introduction}

Massive stars play key roles in various astrophysical contexts all along their evolution: they ionise ultra-compact HII regions while still embedded in their parental molecular cloud; they create ionised cavities and shape the surrounding interstellar

Send offprint requests to: F. Martins,

e-mail: fabrice.martins@obs.unige.ch

* Appendix A is only available in electronic form at http://www.edpsciences.org medium during the main fraction of their lifetime; they experience strong episodes of mass loss when they become Luminous Blue Variables and Wolf-Rayet stars, revealing their core and enriching the ISM in products of $\mathrm{H}$ and He burning; they end their life as supernovae, producing the heavy elements and releasing large amounts of mechanical energy. During all these phases, massive stars lose mass through winds driven by radiation pressure on metallic lines. This affects not only their evolution (e.g., Chiosi \& Maeder 1986) but also the surrounding interstellar medium in which the release of mechanical 
energy can trigger instabilities leading to the collapse of molecular clouds and to star formation. Moreover, bubbles and superbubbles observed on galactic scales are powered by such mass ejections. Hence, various astrophysical fields require the knowledge of quantitative wind properties of massive stars.

Several studies have been carried out in the last two decades to determine these properties. At solar metallicity, the observational determinations (e.g., Howarth \& Prinja 1989; Puls et al. 1996; Herrero et al. 2000) are on average in good agreement with the most recent hydrodynamical predictions based on the radiation driven wind theory (Vink et al. 2000), both in terms of mass loss rate and of the modified wind momentumluminosity relation (WLR; e.g., Puls et al. 1996) which quantifies the strength of the wind. At nonsolar metallicities, we expect the wind properties to vary with $Z$ due to the modified radiative acceleration through metallic lines. In particular, the mass loss rate should be proportional to $Z^{r}$ (Abbott 1982; Puls et al. 2000) and the WLR should be shifted towards lower values and should have a steeper slope. The most recent theoretical results predict $r \sim 0.8$ (Vink et al. 2001) but no change in the slope of the WLR, at least for $Z>10^{-3} Z_{\odot}$ (Hoffmann et al. 2002; Kudritzki 2002). Observational studies indicate a reduction of the mass loss rate and of the terminal velocity in the Magellanic Clouds, but given the small number of objects studied so far, the behaviour of the WLR at low metallicity is still poorly understood. Several groups are currently analysing stars in subsolar (Crowther et al. 2002; Hillier et al. 2003; Bouret et al. 2003) and supersolar (Najarro et al. 1997; Figer et al. 2002) regions for a better understanding of wind properties in different environments. The present work on SMC-N81 stars takes part in this effort.

The SMC-N81 region belongs to the class of the "High Excitation Blobs" (HEB) first introduced by Heydari-Malayeri $\&$ Testor (1982). These blobs are compact regions of star formation in the Magellanic Clouds (see Heydari-Malayeri (2002b) for a complete review). They have a typical radius of a few $\mathrm{pc}$ and display the features of starforming regions: HII cavities, turbulent structures, ionisation fronts and shocks. Recent HST observations (Heydari-Malayeri et al. 1999) have revealed for the first time its stellar content, 4 of the brightest stars being grouped in the central $2^{\prime \prime}$ wide region. Subsequently spectra of the main exciting stars have been obtained with STIS onboard HST. The qualitative analysis of these spectra, presented in Heydari-Malayeri et al. (2002a, hereafter Paper I), has already revealed interesting properties. First, the stars have been identified as mid $\mathrm{O}$ dwarfs with surprisingly low luminosities compared to "classical" dwarfs. Second, the UV spectra have shown signatures of very weak winds, even weaker than those usually observed in the SMC. These characteristics have led Heydari-Malayeri et al. (2002a) to propose that the SMC-N81 stars could belong to the class of Vz stars, which are massive stars thought to lie very close to the ZAMS (Walborn \& Parker 1992).

As such the properties of these stars, showing unusually weak winds compared to other SMC O stars, seem already quite interesting. Furthermore the association of these objects with a compact star forming region, presumably indicative of a very young age, also allows one to obtain unique constraints on properties of very young massive stars shortly after their birth. In fact such observations appear crucial for a better understanding of the earliest evolutionary phases of massive stars and for constraining their formation process which is still under debate (they may form by accretion on a protostellar core - Norberg \& Maeder 2000; Behrend \& Maeder 2001 - or by collisions between low mass components in dense stellar clusters - Bonnell et al. 1998).

With such objectives in mind we have carried out a quantitative study of the UV spectra of the SMC-N81 stars. First results have been presented in Martins et al. (2002a). In fact we are able to determine upper limits on the mass loss rates of four $\mathrm{O}$ stars in this region, which turn out to be surprisingly low (typically $\dot{M} \lesssim$ a few $10^{-9} M_{\odot} \mathrm{yr}^{-1}$ ) compared to predictions of the radiation driven wind theory, even when taking metallicity effects into account. Although no precise physical explanation is found for this behaviour we strongly suggest that this behaviour is related to the very youth of these massive stars.

The remainder of the paper is structured as follows. Section 2 briefly summarises the observations and data reduction. Section 3 describes the main ingredients of the modeling. In Sect. 4 we explain how interstellar lines are taken into account. The main results are given in Sect. 5 and discussed in Sects. 6 (nebular and stellar properties) and 7 (wind properties). Finally, Sect. 8 summarises the main results.

\section{Observations and data reduction}

Ten SMC N81 stars were observed with STIS onboard HST on 28 and 31 October 1999 (General Observer Program No 8246, PI M. Heydari-Malayeri). The spectra in the wavelength range 1120-1715 $\AA$ were obtained through the G140 L grating on the Multi-Anode Microchannel Array (MAMA) detector. The $52^{\prime \prime} \times 0.2^{\prime \prime}$ entrance slit was used. The effective resolution was $0.6 \AA$ per pixel of $25 \mu \mathrm{m}$ which corresponds to a dispersion of $24 \AA \mathrm{mm}^{-1}$ or a resolution of $1.2 \AA$. The exposure times were chosen to equalize the $S / N$ ratios, which are of the order 20 for the 4 stars studied here. The other stars have lower $S / N$ ratio which precludes any quantitative analysis. Optical spectra, also obtained with STIS for 6 objects, were of insufficient quality (too low spectral resolution) and are therefore not used here.

Details concerning the data reduction can be found in Paper I.

\section{CMFGEN: Basic concepts}

The modeling of realistic atmospheres of massive stars requires the inclusion of three main ingredients: 1) due to the high luminosity of these stars, radiative processes are dominant and a non-LTE treatment must therefore be done; 2) mass loss creates an atmosphere which can extend up to several tens of stellar radii which renders the use of spherical geometry unavoidable; 3 ) the inclusion of metals (mostly iron) is fundamental to reproduce realistic atmospheric structures and emergent spectra (line-blanketing effect).

We are now in an area where powerful tools including most of the above ingredients with progressively fewer 
approximations are becoming available. Examples are the codes TLUSTY (Hubeny \& Lanz 1995), WM-BASIC (Pauldrach et al. 2001) and FAST-WIND (Santolaya-Rey et al. 1997). For our study, we have chosen to use the program CMFGEN (Hillier \& Miller 1998) now widely used for spectroscopic studies of massive stars. The main ingredients taken into account in CMFGEN are the following:

- non-LTE approach: the whole set of statistical equilibrium equations and radiative transfer equations is solved to yield the level populations and the radiative field;

- wind extension: all equations are written in spherical geometry with the assumption of spherical symmetry and include all velocity terms due to the expanding atmosphere;

- line-blanketing: metals are included in the statistical equilibrium equations so that accurate level occupation numbers can be derived. A super-level approximation consisting in gathering levels of close energy into a unique super-level is used to reduce the computational cost;

- radiative equilibrium: the temperature structure in the atmosphere is set by the condition of radiative equilibrium. Other heating/cooling sources are optional and can be included (adiabatic cooling, X-rays);

- hydrodynamical structure: at present, CMFGEN does not compute the velocity and density structure so that they have to be taken as input data or have to be parameterised. In our approach, they are computed by other atmosphere codes. We use either the ISA-WIND code which uses the hydrodynamical equations together with a grey LTE temperature to yield the density and velocity in the atmosphere (see also Martins et al. 2002b), or TLUSTY which, thanks to a more accurate description of the pressure terms, gives a good description of the photospheric structure which is connected to a wind velocity structure represented by a classical $\beta$ law $\left(v=v_{\infty}\left(1-\frac{R_{\star}}{r}\right)^{\beta}\right)$. Tests made with stellar and wind parameters typical of our stars have shown that the two methods give similar results for dwarfs in terms of emergent UV spectra.

Several input parameters have to be specified, the main ones being:

- stellar parameters: luminosity $(L)$, radius $(R)$, mass $(M)$;

- wind parameters: mass loss rate $\left(\dot{M}\right.$, terminal velocity $\left.v_{\infty}\right)$. Note that the slope of the velocity field in the wind (the socalled $\beta$ parameter) is chosen when the velocity structure is computed so it is also an input parameter (with default value 0.8 ). CMFGEN also gives the possibility to include clumping. This is done by the inclusion of a volume filling factor $f$ of the form $f=f_{\infty}+\left(1-f_{\infty}\right) \mathrm{e}^{-\frac{v}{v_{\text {init }}}}$ where $f_{\infty}$ is the value of $f$ in the outer atmosphere and $v_{\text {init }}$ the velocity at which clumping appears $\left(30 \mathrm{~km} \mathrm{~s}^{-1}\right.$ by default in our computations);

- abundances/elements: in most of our models, the metallicity has been chosen to be $1 / 8$ solar where solar refers to the values by Grevesse \& Sauval (1998). This metallicity is thought to be typical of stars in the SMC and in N81 in particular (Venn 1999; Hill 1999; Vermeij et al. 2002),
Table 1. Ions included in the atmosphere models. Numbers in parentheses indicate species which, for given $T_{\text {eff }}$, are trace ions and then are not taken into account.

\begin{tabular}{|c|c|c|c|c|c|c|c|c|}
\hline Element & & \multicolumn{7}{|c|}{ Ionisation state } \\
\hline $\mathrm{H}$ & I & II & & & & & & \\
\hline $\mathrm{He}$ & I & II & III & & & & & \\
\hline $\mathrm{C}$ & & (II) & III & IV & V & & & \\
\hline $\mathrm{N}$ & & (II) & III & IV & $\mathrm{V}$ & VI & & \\
\hline $\mathrm{O}$ & & (II) & III & IV & $\mathrm{V}$ & VI & (VII) & \\
\hline $\mathrm{Si}$ & & (II) & (III) & IV & $\mathrm{V}$ & & & \\
\hline S & & & (III) & IV & $\mathrm{V}$ & VI & (VII) & \\
\hline $\mathrm{Fe}$ & & & (III) & IV & $\mathrm{V}$ & VI & VII & (VIII) \\
\hline
\end{tabular}

although the exact value remains uncertain. The ions included in our computations are given in Table 1;

- turbulent velocity: a value of $20 \mathrm{~km} \mathrm{~s}^{-1}$ was chosen for the calculation of the populations and of the temperature structure. Martins et al. (2002b) have shown that reasonable changes of $v_{\text {turb }}$ had few effects on this part of the calculation. For the formal solution of the radiative transfer equation leading to the final detailed emergent spectrum, the value of $v_{\text {turb }}$ has been determined to give the best fit as shown in Sect. 5.5 and is found to be of the order of $5 \mathrm{~km} \mathrm{~s}^{-1}$.

\section{Interstellar lines}

The determination of mass loss rates relies on the fit of emission or P-Cygni lines (see Sect. 5.2). The synthetic profiles are quite sensitive to the value of $\dot{M}$, so that a reliable estimate of this quantity requires the best possible knowledge of the stellar and wind spectrum. Consequently, the contaminating interstellar (IS) lines must be identified, which is easy in high resolution spectra where these IS lines appear as narrow absorptions in the P-Cygni profiles. However, in our medium resolution observations the IS components are diluted in the stellar features so that the exact stellar + wind profiles are uncertain. Of course, this depends on the line: as $\mathrm{N} \mathrm{V}$ is a trace ion in the interstellar medium, stellar N v $\lambda 1238,1242$ is weakly affected by this problem. But this is not the case for C IV $\lambda \lambda 1548,1551$ which is all the more contaminated given that the wind feature is weak in the observed N81 spectra. It is therefore crucial to estimate the contribution of the interstellar absorption to derive reliable mass loss rates.

The interstellar absorption originates both in the Galaxy and the local SMC environment. To estimate the interstellar C IV column densities, we have proceeded as follows:

- First, we have used high resolution HST-STIS spectra of 8 SMC stars (AV 69, AV 75, AV 80, AV 327, NGC 346 355, NGC 346 368, NGC 346 113, NGC 346 12), for which UV spectra have been obtained from the HST archive, to determine the C IV column densities in the direction of the NGC 346 region and the southwest part of 
Table 2. Determination of CIV column densities.

\begin{tabular}{lll}
\hline \hline Component & $\log (N(\mathrm{C}$ IV $))$ & Reference \\
\hline Galactic & $14.4 / 14.5_{-0.10}^{+0.10}$ & Mallouris et al. (2001) \\
& 14.4 & Fitzpatrick \& Savage (1983) \\
& $14.35_{-0.06}^{+0.06}$ & Sembach \& Savage (1992) \\
& $14.28_{-0.13}^{+0.10}$ & SMC stars, this work \\
SMC & $14.4 / 14.5_{-0.10}^{+0.10}$ & Mallouris et al. (2001) \\
& $>14.5$ & Fitzpatrick \& Savage (1983) \\
& $14.43_{-0.40}^{+0.20}$ & SMC stars, this work \\
\hline
\end{tabular}

the SMC. For that purpose, the Galactic and SMC interstellar profiles have been fitted with a Gaussian profile (with a shift of $140 \mathrm{~km} \mathrm{~s}^{-1}$ - the receding velocity - for the SMC component). Two parameters are needed to achieve such a fit: the column density and the FWHM of the Gaussian profile (for which a typical value of $20 \mathrm{~km} \mathrm{~s}^{-1}$ was chosen). The mean column densities derived from this study are the following: $\log (N(\mathrm{C}$ IV $))=14.28_{-0.13}^{+0.10}$ for the Galactic component, and $\log (N(\mathrm{C}$ IV $))=14.43_{-0.40}^{+0.20}$ for the SMC component. The higher dispersion in the case of the SMC component is probably due to the fact that we are looking at different parts of the SMC, whereas only one line of sight is used for the Galaxy.

- Second, we have taken various determinations of the C IV column density from the literature. Several methods were used (curve of growth, line fitting, apparent optical depth) and give consistent results.

Table 2 summarises the various column density estimates. For the Galactic case, the results from our determination are consistent with more accurate determinations (within the errors). For subsequent analysis, we adopt $\log (N(\mathrm{C}$ IV $)=14.4$ as a representative value for the Galactic absorption. For the SMC, the results are also in good agreement. As the reddening of the SMC-N81 stars is similar to that of the other 8 SMC stars used for this study (the values of $E(B-V)$ are of the order of 0.12 for all stars), the properties of the interstellar matter on the different lines of sight towards the SMC sampled here must be the same. This absence of local extinction was noted by Heydari-Malayeri et al. (1988) in the first detailed study of SMC-N81. Then the value of $\log (N(\mathrm{C}$ IV $)=14.5$ derived on average for the SMC regions in Table 2 is chosen to be typical of the CIV column density in the direction of N81.

With these column densities, we have created synthetic profiles of the interstellar C IV absorption lines. The method used was the same as that employed to estimate the column densities from the spectra of the 8 SMC stars mentioned above. We have then added these interstellar contributions to the CMFGEN profiles. Figure 1 shows an example of such a correction. The typical uncertainties in the column densities translate to modifications of the depth of the corrected absorption profile of the order of 0.05 .

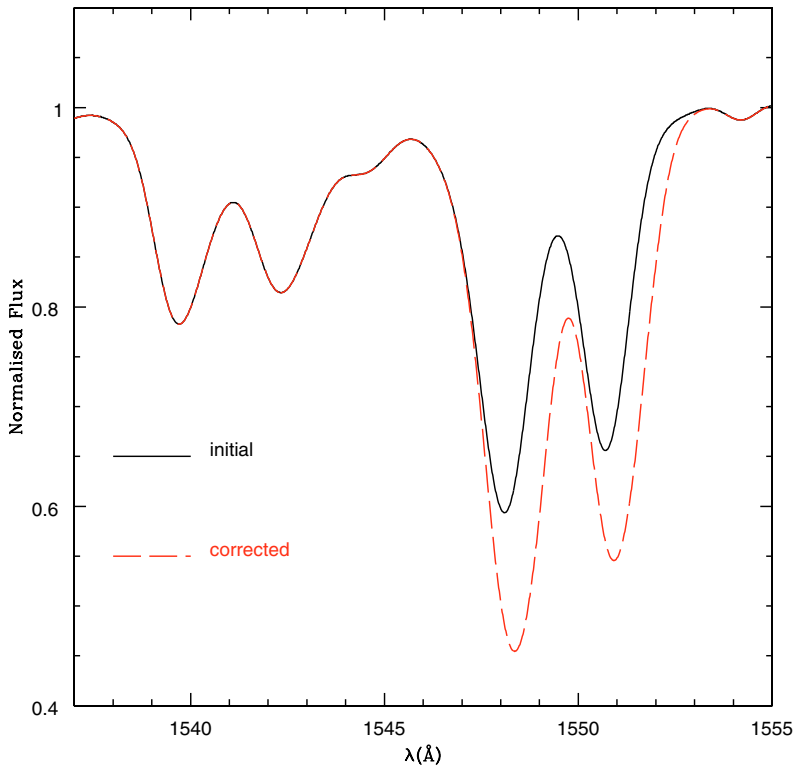

Fig. 1. Interstellar C IV component addition: the black solid line shows the C IV $\lambda \lambda 1548,1551$ profile of a CMFGEN model, and the red long dashed line is the resulting profile after including the IS absorption. A convolution has been performed to take into account the instrumental resolution $(1.2 \AA)$. The parameters used to model the interstellar component are: $\log \left(N(\mathrm{C} \text { IV })_{\text {gal }}\right)=14.4, \log \left(N(\mathrm{C} \text { IV })_{\text {SMC }}\right)=14.5$ and $v_{\mathrm{SMC}}=140 \mathrm{~km} \mathrm{~s}^{-1}$.

\section{Detailed analysis of individual stars}

In this section, we present the results of the quantitative analysis of the UV spectra of our target stars. Constraints on the effective temperature, the luminosity, the terminal velocity of the wind and the mass loss rate constitute the main outputs. Secondary constraints on the slope of the velocity field or the amount of clumping are also given. The method used is explained in detail for the case of star 2, while for the other stars the results are summarised in Sect. 5.7 and in Table 4.

\subsection{Effective temperature}

The most reliable $T_{\text {eff }}$ diagnostics for $\mathrm{O}$ stars remain the photospheric helium lines in the optical. Unfortunately, optical spectra of the SMC-N81 stars are not available so that we had to rely on the UV. Two types of indicators were used in this spectral range: the shape of the SED and the strength of several lines.

\subsubsection{UV colour index}

As O stars emit most of their luminosity in the UV, the shape of the spectral energy distribution at these wavelengths is sensitive to the effective temperature just as is the optical spectrum in the case of cooler stars. A colour index in the UV can then make it possible to determine $T_{\text {eff }}$. This has been done observationally by Fanelli et al. (1992) who have computed various spectral indices based on IUE spectra for different groups of stars.

We have used the recent grid of $\mathrm{O}$ dwarf models by Martins et al. (2002b) recomputed for an SMC metallicity $\left(Z=1 / 8 Z_{\odot}\right)$ completed by various models at this low $Z$ to derive a relation 


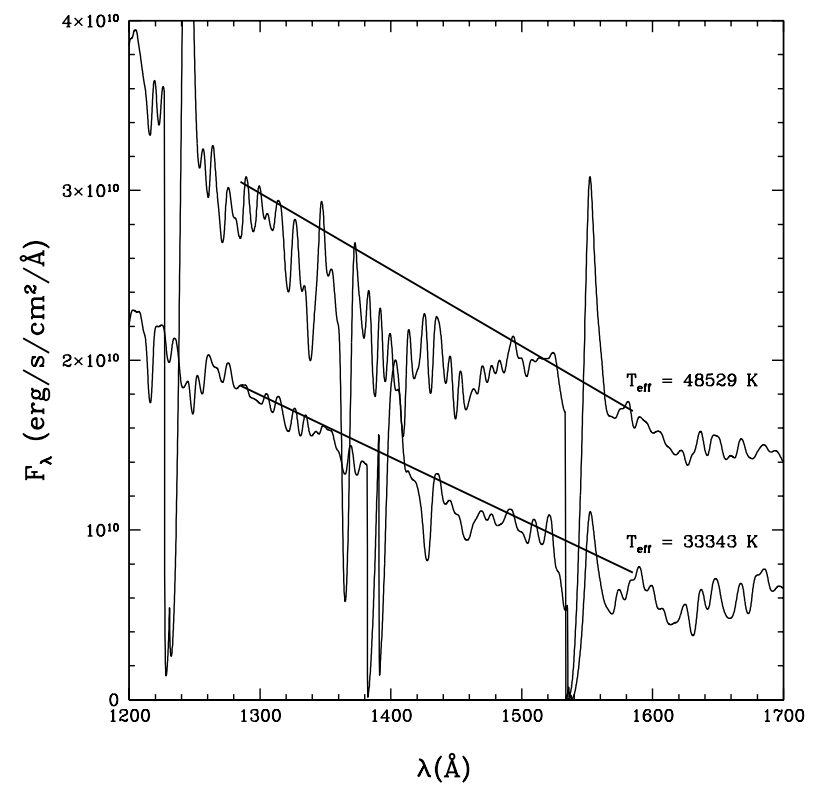

Fig. 2. UV flux distribution of two O dwarf models at $33343 \mathrm{~K}$ and $48529 \mathrm{~K}$. Whereas the slope $F_{1285}-F_{1585}$ increases with $T_{\text {eff }}$ the ratio $\frac{F_{1285}}{F_{1585}}$ decreases. The bold lines are to guide the eye and to show the variations of the slope.

between effective temperature and a synthetic colour index defined by $\frac{F_{1285}}{F_{1585}}$ where $F_{1285}\left(F_{1585}\right)$ is the mean flux in an artificial $20 \AA$ wide box-shaped filter centered on $1285 \AA$ (1585). The choice of these wavelengths was a compromise between having fluxes in distant wavelength ranges (to get a ratio significantly different from 1 ) and avoiding metallicity effects (see below). In the UV part of the spectrum of interest to us, an increase of $T_{\text {eff }}$ translates to a decrease of $\frac{F_{1285}}{F_{1585}}$ (whereas the slope of the spectrum, $F_{1285}-F_{1585}$, increases). This is illustrated in Fig. 2 where we see that an increase of $T_{\text {eff }}$ from $33343 \mathrm{~K}$ to $48529 \mathrm{~K}$ induces a decrease of $\frac{F_{1285}}{F_{1585}}$ from $\sim 2.5$ to $\sim 1.8$ while the slope increases (see the bold lines). This is confirmed observationally by Fanelli et al. (1992).

Figure 3 shows the correlation between our UV colour index and $T_{\text {eff. }}$ The determination of the UV colour index from the dereddened flux distribution of the SMC-N81 stars together with this theoretical relation allows us to estimate $T_{\text {eff }}$ of the observed stars. In the case of star 2 , we found a value of $\sim 37500 \mathrm{~K}$.

This method suffers from various problems. The most important is probably the extinction which modifies the slope of the SED and renders the UV colour index uncertain. The dashed line in Fig. 3 shows the position of star 2 if its SMC extinction was increased by 0.02 (compared to the extinction derived from photometry): in that case, the estimated $T_{\text {eff }}$ would be $\sim 34000 \mathrm{~K}$. This may seem surprising, as naively a higher $T_{\text {eff }}$ could be expected for a higher extinction. However, this behaviour is simply due to the fact for a "normal" extinction law (e.g., Prévot et al. 1984) an increase of $E(B-V)$ translates into an increase of $F_{\text {dered }}^{1285} / F_{\text {dered }}^{1585}$ which corresponds to a lower $T_{\text {eff }}$ as discussed above. Figure 3 shows that a typical error of 0.02 on $E(B-V)$ (i.e. an error on the flux determination)

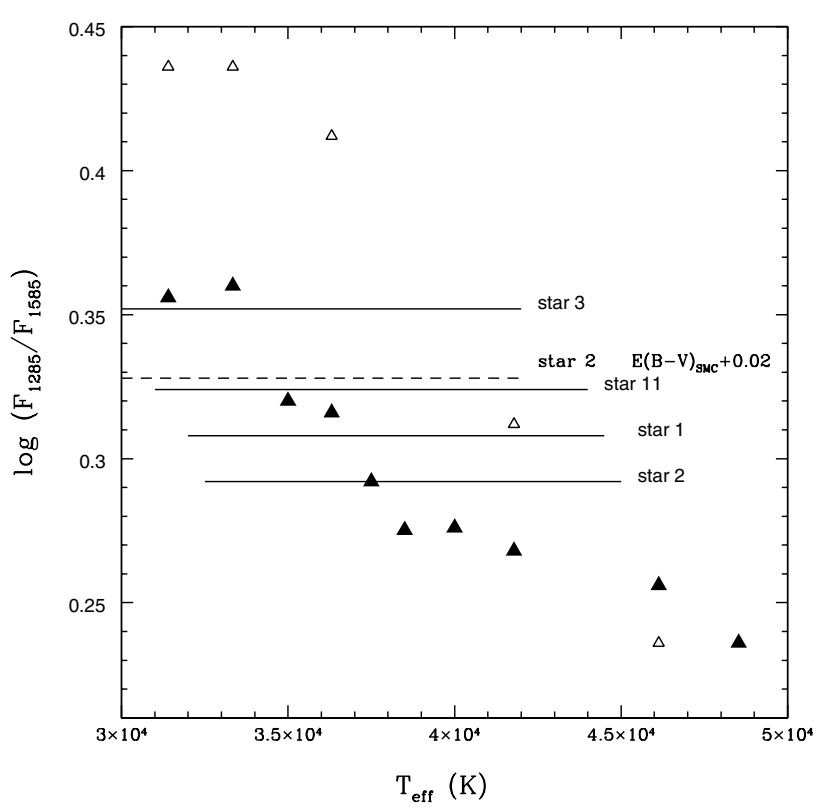

Fig. 3. Effective temperature indicator: colour index method. The colour index is define by $\frac{F_{1285}}{F_{1585}}$ where $F_{1285}\left(F_{1585}\right)$ is the mean flux at $1285 \AA$ (1585 $\AA$ ). Filled (open) triangles are for models with $Z=$ $1 / 8 Z_{\odot}\left(Z=Z_{\odot}\right)$. Horizontal lines indicate the colour index for N81 stars which, by comparisons with the theoretical values, give an estimate of the effective temperature. Note that this method is strongly metallicity-dependent. The dashed line show the position of star 2 for an SMC extinction increased by 0.02 .

translates to an uncertainty of the order of $3000 / 4000 \mathrm{~K}$ in $T_{\text {eff }}$ based on the UV colour index method.

The UV SED is also shaped by many metallic (mostly iron) lines and thus metallicity can affect the determination of UV magnitudes. This is clearly demonstrated in Fig. 3 where the effect of increasing the metallicity from $1 / 8 Z_{\odot}$ to $Z_{\odot}$ strongly steepens the slope of the relation UV colour index $-T_{\text {eff. }}$ Both the slope of the continuum and the "line forests" are responsible for such a behaviour. Our choice of filters centered at $1285 \AA$ and $1585 \AA$ (where the density of metallic lines is weaker than in other wavelength ranges) was made to try to minimise the latter effect.

Finally, the slope of the SED also depends on the gravity (e.g., Abbott \& Hummer 1985). However, for dwarf stars, this dependence is weak: a test case run for a model with $\log g=4.1$ and 4.4 has shown a change of the ratio $\frac{F_{1285}}{F_{1585}}$ of less than $2 \%$.

Given the above uncertainties, the UV colour index method can only give an indication of the effective temperature which needs to be confirmed by other indicators.

\subsection{2. spectral lines}

Several spectral features can be used as $T_{\text {eff }}$ indicators in the UV.

\section{- O IV $\lambda \lambda 1339,1343 / \mathrm{O}$ v $\lambda 1371$ :}

O IV $\lambda \lambda 1339,1343$ is present in the spectra of most $O$ dwarfs while O v $\lambda 1371$ appears only in stars earlier than O6 (Walborn et al. 1995) so that their presence/absence and relative strength 

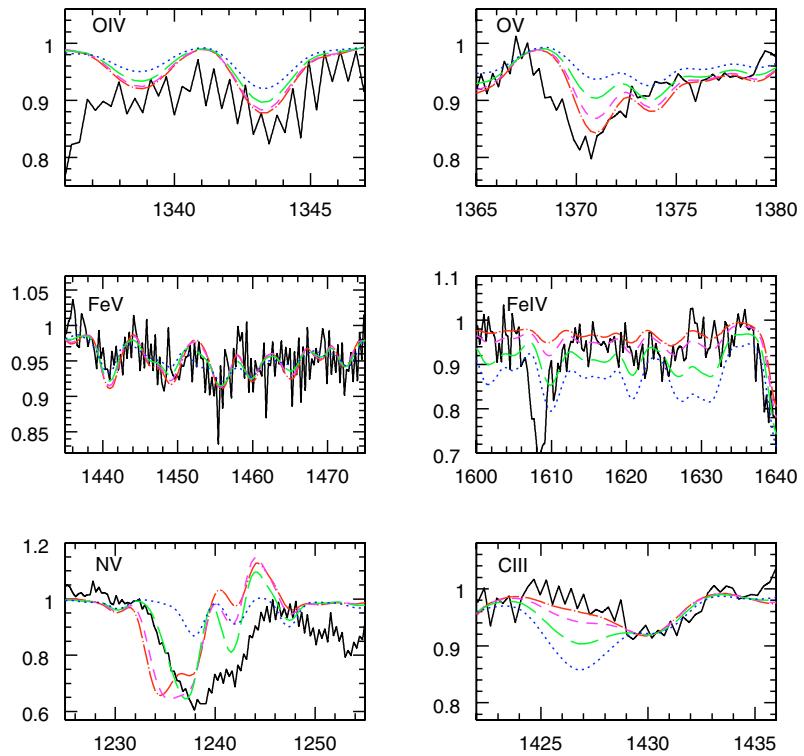

Fig. 4. Spectroscopic $T_{\text {eff }}$ indicators. The black solid line is the observed spectrum of star 2 . Coloured lines are four different models with $T_{\text {eff }} 42000 \mathrm{~K}$ (red, dot-long dashed line), $40000 \mathrm{~K}$ (magenta, short dashed line), $37500 \mathrm{~K}$ (green, long dashed line), $35000 \mathrm{~K}$ (blue, dotted line). The mass loss rate is fixed at a constant value of $10^{-8.5} M_{\odot} \mathrm{yr}^{-1}$. See text for discussion.

is a good $T_{\text {eff }}$ estimator. Figure 4 shows comparisons between models of different $T_{\text {eff }}$ and the observed spectrum of star 2. An increase of $T_{\text {eff }}$ increases the strength of both lines which are reproduced for $T_{\text {eff }} \geq 40000 \mathrm{~K}$.

O v $\lambda 1371$ alone has been used by de Koter et al. (1998) as a $T_{\text {eff }}$ indicator for early $\mathrm{O}$ stars. Nonetheless, as mentioned by these authors, this line is always predicted too strong compared to observations above $\sim 40000 \mathrm{~K}$. Below this limit, the situation seems better. Recently, Bouret et al. (2003) have claimed that clumping could help to solve this well known problem (see Schaerer et al. 1996; Pauldrach et al. 2001): the line is weaker in a clumped model than in a homogeneous wind. As O v $\lambda 1371$ (together with O IV $\lambda \lambda 1339,1343$ ) depends also on $\dot{M}$, our $T_{\text {eff }}$ estimate relies on the relative strength of both lines in homogeneous winds. This estimate must be confirmed by stronger indicators.

\section{- $\mathrm{Fe} \mathrm{IV} / \mathrm{Fe} \mathrm{V}$ :}

Iron line forests exist all over the UV range. In particular, Fe IV lines are present between $1600 \AA$ and $1640 \AA$, while Fe V lines are found between $1430 \AA$ and $1480 \AA$. The iron ionisation increases with $T_{\text {eff }}$ so that Fe IV lines weaken relatively to Fe V lines between 35000 and $42000 \mathrm{~K}$. As Fe V is the dominant ionisation state of $\mathrm{Fe}$ in this temperature range, Fe $\mathrm{V}$ lines are saturated and little affected by an increase of $T_{\text {eff }}$ whereas Fe IV lines weaken. This is shown in Fig. 4. For star 2, a value of at least $40000 \mathrm{~K}$ is necessary to reproduce the observed spectrum.

The determination of $T_{\text {eff }}$ from this line ratio can be hampered mainly by two effects:

- the iron abundance (and more generally metallicity) can change the strength of the Fe absorption. This effect is twofold. First, increasing the iron abundance will immediately increase the absorption of all $\mathrm{Fe}$ ions, although differently depending on the position of the lines on the curve of growth. Consequently, the ratio of lines from two successive ionisation states will be modified. Second, increasing the iron abundance will strengthen the line-blanketing effect and thus will increase the local temperature in the line formation region. The ionisation will be increased, leading to a higher $T_{\text {eff }}$ estimate. The effects of metallicity due to line-blanketing on the effective temperature of $\mathrm{O}$ stars have been estimated by Martins et al. (2002b) and turn out to be of the order of 1000 to $2000 \mathrm{~K}$ depending on the spectral type for metallicities ranging from solar to $1 / 8$ solar. We have run test models for a global metallicity of $Z=1 / 5 Z_{\odot}$. Its main effect is to strengthen the Fe IV lines, leaving the $\mathrm{Fe} \mathrm{V}$ lines unchanged as they are almost saturated in the temperature range of interest here. Quantitatively, this change of $Z$ is equivalent to a decrease of $T_{\text {eff }}$ by $\sim 2000 \mathrm{~K}$ as regards the iron lines behaviour;

- the so-called turbulent velocity "artificially" strengthens the absorption profiles as shown in Fig. 12 where $v_{\text {turb }}$ increases from 5 to $15 \mathrm{~km} \mathrm{~s}^{-1}$. Fe IV and $\mathrm{Fe} \mathrm{V}$ lines deepen differently when $v_{\text {turb }}$ increases, so that their relative strength remains turbulent-velocity dependent. Nonetheless, the effect is smaller than the effect on individual lines so that a reasonable estimate of $T_{\text {eff }}$ can be drawn from the study of the relative strength of these iron forests. Note that if the effective temperature is known, the iron lines can be used to determine the turbulent velocity (see Sect. 5.5).

\section{- C III $\lambda \lambda 1426,1428$ :}

As noted by Walborn et al. (1995) in their IUE atlas of O star spectra, this blend of C III lines strengthens towards later types. Figure 4 shows that in the case of star 2 a value of at least $42000 \mathrm{~K}$ is required to fit the observed spectrum. Changing slightly the carbon content can affect this determination. Quantitatively, a reduction of the $\mathrm{C}$ abundance from $1 / 8 C_{\odot}$ to $1 / 10 C_{\odot}$ (as discussed in Sect. 5.2.2) is equivalent to an increase of $T_{\text {eff }}$ by $\sim 1500 \mathrm{~K}$.

\section{- $\mathrm{N}$ v $\lambda 1238,1242$ :}

This resonance line is known to be strongest around spectral type O4 (Smith-Neubig \& Bruhweiler 1997) and to weaken at later spectral types or equivalently when $T_{\text {eff }}$ decreases. Quantitatively, a $T_{\text {eff }}>35000 \mathrm{~K}$ is required to account for the $\mathrm{N}$ V $\lambda 1238,1242$ absorption profile in star 2. Nonetheless, the strong mass loss dependence of this line makes it a poor and only secondary $T_{\text {eff }}$ indicator: a high mass loss associated with a relatively low $T_{\text {eff }}$ can mimic the $\mathrm{Nv}$ profile of a star with a lower mass loss rate but a higher $T_{\text {eff }}$.

Table 3 summarises the results of these $T_{\text {eff }}$ estimates, which all point to an effective temperature of the order $40000 \mathrm{~K}$ for star 2. A real dispersion exists and is mostly due to the multi-parameter dependence of most of the indicators used. 
Table 3. Effective temperature estimates.

\begin{tabular}{|c|c|c|c|}
\hline Star & Estimator & $T_{\text {eff }}(\mathrm{K})$ & Adopted $T_{\text {eff }}(\mathrm{K})$ \\
\hline \multirow[t]{6}{*}{1} & UV colour index & 37000 & \\
\hline & OIV/OV & 36000 & \\
\hline & $\mathrm{Fe} \mathrm{IV} / \mathrm{Fe} \mathrm{V}$ & $\gtrsim 38500$ & \\
\hline & $\mathrm{N} \mathrm{v}$ & - & \\
\hline & C III & 38500 & \\
\hline & & & 38500 \\
\hline \multirow[t]{6}{*}{2} & UV colour index & 37500 & \\
\hline & $\mathrm{O} \mathrm{IV} / \mathrm{O} \mathrm{V}$ & $\gtrsim 40000$ & \\
\hline & $\mathrm{Fe} \mathrm{IV} / \mathrm{Fe} \mathrm{V}$ & 40000 & \\
\hline & $\mathrm{N} \mathrm{v}$ & $>35000$ & \\
\hline & C III & 42000 & \\
\hline & & & 40000 \\
\hline \multirow[t]{6}{*}{3} & UV colour index & 34000 & \\
\hline & $\mathrm{O} \mathrm{IV} / \mathrm{O} \mathrm{V}$ & $\lesssim 37500$ & \\
\hline & $\mathrm{Fe} \mathrm{IV} / \mathrm{Fe} \mathrm{V}$ & 36000 & \\
\hline & $\mathrm{N} \mathrm{v}$ & $<37500$ & \\
\hline & C III & 36000 & \\
\hline & & & 36000 \\
\hline \multirow[t]{6}{*}{11} & UV colour index & 35000 & \\
\hline & $\mathrm{O}$ IV/O v & 37000 & \\
\hline & $\mathrm{Fe} \mathrm{IV} / \mathrm{Fe} \mathrm{V}$ & $\gtrsim 37000$ & \\
\hline & $\mathrm{N} \mathrm{v}$ & $<37000$ & \\
\hline & C III & 37000 & \\
\hline & & & 37000 \\
\hline
\end{tabular}

\subsubsection{Estimation of uncertainty on $T_{\text {eff }}$}

Table 3 shows that for a given star the dispersion in the $T_{\text {eff }}$ estimators is of the order of $\pm 2500 \mathrm{~K}$ in the worst cases. A possible additional source of uncertainty comes from the rectification of the spectra. We estimate it to be lower than 0.05 in terms of normalised flux, which from Fig. 4 (especially the FeV plot) can lead to an error on $T_{\text {eff }}$ of the order of $\pm 1500 \mathrm{~K}$.

The uncertainties due to variations of the global metallicity and carbon content have been estimated above and are of the order of $2000 \mathrm{~K}$.

To estimate the efficiency of our $T_{\text {eff }}$ determination method, we have applied it to a star for which optical and UV analysis have given strong constraints on $T_{\text {eff. }}$ For this purpose, $10 \mathrm{Lac}$ was chosen (see Sect. 7.2). The UV colour index method indicates a value of $39000 \mathrm{~K}$, while the spectral lines point to values of the order $35000 / 36000 \mathrm{~K}$. As the accepted $T_{\text {eff }}$ for $10 \mathrm{Lac}$ is close to $36000 \mathrm{~K}$, the error on the $T_{\text {eff }}$ estimate is not more than $\pm 3000 \mathrm{~K}$. This example also shows that the spectral line method is more accurate than the colour index method. For that reason we have given more weight to the $T_{\text {eff }}$ estimates based on the line method in our final estimate (as can be shown in Table 3).
From the above discussion, we conclude that on average, the uncertainty on our $T_{\text {eff }}$ determination is of the order of $\pm 3000 \mathrm{~K}$.

\subsection{Mass loss rate}

The determination of the mass loss rate remains one of the main goals of this study. As already noted, the spectra of N81 stars show very weak wind features with no emission. As emission, contrary to absorption, is entirely formed in the wind, our constraints on $\dot{M}$ have been set by the requirement that no emission is produced in the model spectra (as observed in the STIS spectra). Thus, we have derived only upper limits on the mass loss rates as models with $\dot{M}$ below this limit never produce emission. The main mass loss indicators in the UV are the $\mathrm{N}$ v $\lambda 1238,1242, \mathrm{C}$ IV $\lambda \lambda 1548,1551$ and $\mathrm{O} v \lambda 1371$ lines.

\subsubsection{Primary determinations}

- O v $\lambda 1371$ :

O V $\lambda 1371$ develops a P-Cygni profile in the earliest supergiants while only an absorption is seen in dwarfs (Walborn et al. 1995). Figure 5 reveals that the profile deepens when $\dot{M} \geq 10^{-8} M_{\odot} \mathrm{yr}^{-1}$ but remains roughly unchanged below this value. Fitting the observed spectrum of star 2 requires a mass loss rate lower than $10^{-8} M_{\odot} \mathrm{yr}^{-1}$. As mentioned in the previous section, $\mathrm{O} v \lambda 1371$ is sensitive to $T_{\text {eff }}$ so that a degeneracy $\dot{M} / T_{\text {eff }}$ exists. X-rays can also affect this line by increasing the Oxygen ionisation to produce O VI (observed in the EUV) by Auger ionisation of O IV.

- $\mathrm{N}$ v $\lambda 1238,1242$ :

This line is present in dwarfs of spectral type earlier than $\mathrm{O} 8$ (Walborn et al. 1995; Smith-Neubig \& Bruhweiler 1997) and shows a strong P-Cygni profile in early dwarfs. It is mostly formed in the wind so that it is one of the best mass loss rate indicators of the UV part of the spectrum. This is illustrated in Fig. 5 where we see the profile changing from a well developed P-Cygni shape to a weak absorption when $\dot{M}$ is reduced by two orders of magnitudes. A mass loss rate lower than $10^{-9} M_{\odot} \mathrm{yr}^{-1}$ is necessary to produce no emission. However, in that case the absorption profile is too weak. Increasing $\dot{M}$ by a factor of 3 improves the fit of this absorption part but induces an emission (see Sect. 7 for a discussion of the shape of these wind profiles). Nonetheless, as emission is only produced in the wind whereas absorption can originate both in the wind and in the photosphere, we adopt $\dot{M}=10^{-9} M_{\odot} \mathrm{yr}^{-1}$ as a reasonable upper limit.

$\mathrm{N}$ v $\lambda 1238,1242$ is also $T_{\text {eff }}$ sensitive as seen in Sect. 5.1.2 so that any error on $T_{\text {eff }}$ can lead to an error on $\dot{M}$. Another problem comes from the X-rays (supposed to be created by shocks in the outer wind, e.g., Owocki et al. 1988; Feldmeier et al. 1997) which can increase the ionisation of nitrogen, thus leading to modifications of the $\mathrm{N} v \lambda 1238,1242$ profile. A very accurate determination of $\dot{M}$ based on N v $\lambda 1238,1242$ would then require the inclusion of X-rays in the models. However, 
Table 4. Summary of the stellar and wind properties derived for the N81 stars. The spectral types are estimated from the optical spectra of the models giving the best UV fits. As we have only lower limits on the terminal velocities, we have adopted $v_{\infty} / v_{\text {esc }}=2.6$ to compute both the modified wind momenta and the theoretical mass loss rates of Vink et al. (2001). The $\mathrm{Si}, \mathrm{S}$ and $\mathrm{Fe}$ abundances are $1 / 8$ solar and $n(\mathrm{He}) / n(\mathrm{H})$ is 0.1 . The gravity adopted for our computation was $\log g=4.0$ as it is typical of dwarf stars (Vacca et al. 1996) and as we have no diagnostics to estimate the value of this parameter.

\begin{tabular}{|c|c|c|c|c|}
\hline & Star 1 & Star 2 & Star 3 & Star 11 \\
\hline$m_{\mathrm{V}}$ & 14.38 & 14.87 & 16.10 & 15.74 \\
\hline$E(B-V)$ & 0.07 & 0.06 & 0.10 & 0.07 \\
\hline Spectral type & $\mathrm{O} 7$ & O6.5 & O8.5 & 07.5 \\
\hline$M_{\mathrm{V}}$ & -4.84 & -4.32 & -3.21 & -3.48 \\
\hline$T_{\text {eff }}[\mathrm{K}]$ & 38500 & 40000 & 36000 & 37000 \\
\hline $\log \left(L / L_{\odot}\right)$ & 5.32 & 5.16 & 4.59 & 4.73 \\
\hline$R / R_{\odot}$ & 10.3 & 7.9 & 5.0 & 6.9 \\
\hline$M / M_{\odot}$ & 32 & 30 & 19 & 21 \\
\hline$V \sin i\left[\mathrm{~km} \mathrm{~s}^{-1}\right]$ & 200 & 300 & 250 & 250 \\
\hline $\log \dot{M}\left[M_{\odot} \mathrm{yr}^{-1}\right]$ & $\lessgtr-8.0$ & $\lessgtr-8.0$ & $\lesssim-8.5$ & $\lesssim-9.0$ \\
\hline$v_{\infty}\left[\mathrm{km} \mathrm{s}^{-1}\right]$ & $\geq 1500$ & $\geq 1800$ & $\geq 300$ & $\geq 600$ \\
\hline$v_{\mathrm{esc}}$ & 1088 & 1203 & 1204 & 1077 \\
\hline $\log \left(\dot{M} v_{\infty} \sqrt{R}\right)$ & $\lesssim 26.76$ & $\lesssim 26.75$ & $\lesssim 26.14$ & $\lesssim 25.67$ \\
\hline$f_{\infty}$ & 0.01 & 0.01 & 1 & 1 \\
\hline $\log Q_{0}\left[\mathrm{~s}^{-1}\right]$ & 48.85 & 48.76 & 47.99 & 48.41 \\
\hline $\log \dot{M}_{\text {Vink }}\left[M_{\odot} \mathrm{yr}^{-1}\right]$ & -6.96 & -7.25 & -8.31 & -8.04 \\
\hline $\mathrm{C} / \mathrm{C}_{\odot}$ & $1 / 10$ & $1 / 8$ & $1 / 10$ & $1 / 10$ \\
\hline $\mathrm{N} / \mathrm{N}_{\odot}$ & $1 / 20$ & $1 / 8$ & $1 / 20$ & $1 / 20$ \\
\hline $\mathrm{O} / \mathrm{O}_{\odot}$ & $1 / 5$ & $1 / 8$ & $1 / 5$ & $1 / 5$ \\
\hline
\end{tabular}

tests have revealed that the inclusion of X-rays does not modify our conclusions. It is also unlikely that X-ray emission is important since any emission in the wind of the SMC-N81 stars seems to be very weak as shown by the UV spectra.

\section{- C IV $\lambda \lambda 1548,1551$ :}

This line is the other strong UV mass loss indicator of $\mathrm{O}$ dwarfs. It is seen in all $\mathrm{O}$ dwarfs with a strength increasing towards early types (Walborn et al. 1995). In Fig. 5 we see that it shifts from a P-Cygni profile to an absorption profile when $\dot{M}$ decreases. The absence of emission, required by the observation, is obtained for $\dot{M} \leq 10^{-7.5} M_{\odot} \mathrm{yr}^{-1}$. Contrary to $\mathrm{N}$ V $\lambda 1238,1242$, a significant absorption profile can remain even when the emission disappears. But this absorption is mainly photospheric and the wind part turns out to be too weak compared to the observed spectra. A change of $T_{\text {eff }}$ does not lead to any improvement (a lower $T_{\text {eff }}$ implies an emission in C IV $\lambda \lambda 1548,1551$ and a too weak $N$ V $\lambda 1238,1242$ line, and a higher $T_{\text {eff }}$ weakens further the C IV $\lambda \lambda 1548,1551$ line).

\subsubsection{Improving the $\dot{M}$ determinations: Effects of $\beta$, clumping, adiabatic cooling and abundances}

One difficulty in fitting the UV lines (especially C IV $\lambda \lambda 1548,1551$ and $\mathrm{N}$ V $\lambda 1238,1242)$ is to produce a
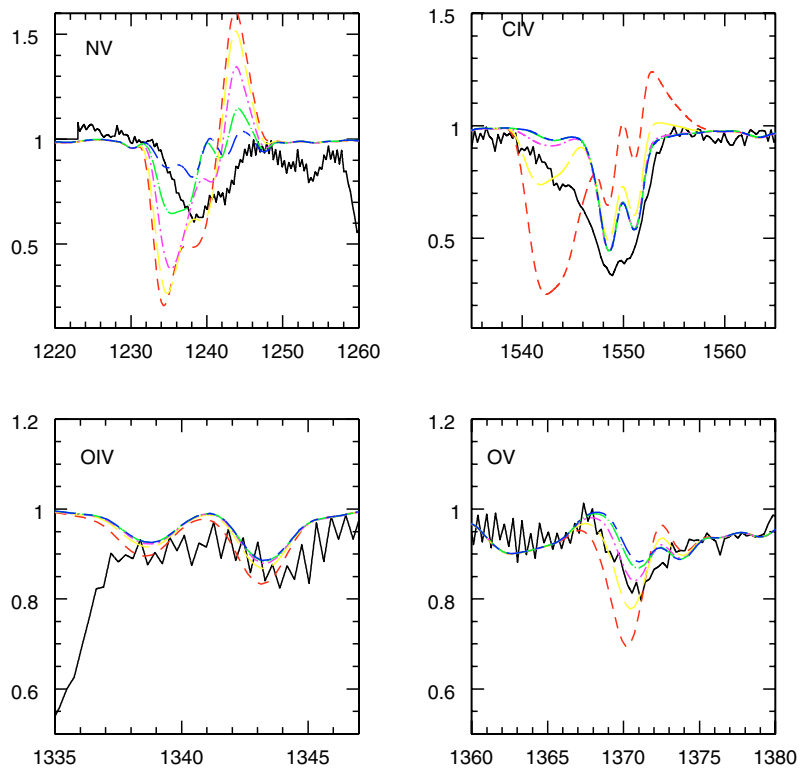

Fig. 5. Mass loss rate indicators: the black solid line is the observed profile while coloured lines are taken from models with $T_{\text {eff }}=40000 \mathrm{~K}$ and various $\dot{M}(-\log (\dot{M})=7$ (red, short dashed line), 7.5 (yellow, long dashed line), 8 (magenta, dot-short dashed line), 8.5 (green, dot-long dashed line), 9 (blue, short dashed - long dashed line)). See text for discussion. 
significant absorption extending up to velocities $\geq 1800 \mathrm{~km} \mathrm{~s}^{-1}$ without any emission. In the following, we concentrate on C IV $\lambda \lambda 1548,1551$ as the discrepancy is highest for this line, but the discussion applies equally to $\mathrm{N} V \lambda 1238,1242$.

The absence or weakness of absorption at high velocity in the models showing no emission results from a lack of absorbers (i.e. C IV ions) which may come either from a too low density, and hence from a too low mass loss rate, or from a too high ionisation of carbon ( $\mathrm{CV}$ being the dominant ionisation state in the wind). The first possibility can be ruled out because a higher mass loss rate will produce an emission which is not observed. Relying on the second hypothesis, we have sought for mechanisms that could lead to a reduction of the ionisation in the wind. This can be achieved if recombination rates are increased, i.e. if the density is higher (recombination scales as $\rho^{2}$ ). As the density is given by $\rho=\frac{\dot{M}}{4 \pi r^{2} v f}$ where $f$ is the filling factor ( $f=1$ in an homogeneous atmosphere), a higher density at a given radius can be obtained by either a higher mass loss rate (which is excluded), or a clumped wind $(f<1)$ or a lower velocity which, as $v_{\infty}$ is fixed, implies a softer slope of the velocity field (the $\beta$ parameter). Recombinations can also be increased when the temperature in the outer wind is reduced. Adiabatic cooling may induce such a reduction as in these low density winds it can become an important cooling process. Last, abundances can of course modify the strength of the wind profiles. In the following, we present investigations of the influence of these various parameters on the line profiles and the mass loss rate determinations.

\section{- $\beta$ effects:}

$\beta$ is usually determined through the shape of hydrogen emission lines in the optical range (e.g., Hillier et al. 2003). However, as optical spectra are not available and as the optical lines probably have absorption profiles due to the weakness of the winds, we have no constraints on this parameter. We have thus run test models with $\beta=2.0$ (the default value in all our computations being 0.8). The results are displayed in Fig. 6. C IV $\lambda \lambda 1548,1551$ is not modified since it is almost purely photospheric in the models shown here, while $\mathrm{N} v \lambda 1238,1242$ shows narrower and stronger absorption and emission when $\beta$ is higher. This behaviour can be explained in terms of the size of the interaction region which is the region in which, due to the wind velocity induced doppler shift, a photon can interact with a given line. The size of this region is proportional to the Sobolev length (see Lamers \& Cassinelli 1999) which scales as $(\mathrm{d} v / \mathrm{d} r)^{-1}$ in the inner wind, so that a higher $\beta$ will lead to larger Sobolev length (the acceleration being smaller). The interaction region is then wider which leads to a stronger absorption or emission in the center of the line. In the outer atmosphere, the radial Sobolev length is proportional to $\beta^{-1} r^{2} / v$ (for a $\beta$ velocity law) and smaller for higher $\beta$ so that the emission/absorption are reduced at high velocity ${ }^{1}$. Illustrations of

1 When $\beta$ increases, the transverse Sobolev length, which is proportional to $(v / r)^{-1}$, increases too. However, the decrease of the radial Sobolev length is greater than the increase of the transverse Sobolev length so that globally, the size of the interaction region is reduced.
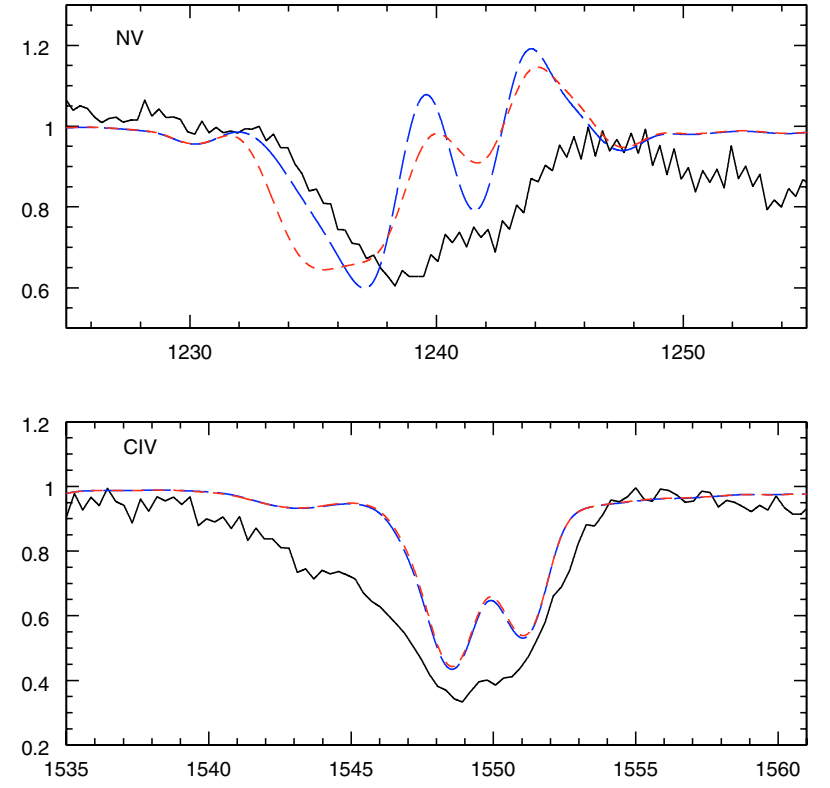

Fig. 6. Influence of the slope of the velocity field $(\beta)$ on the mass loss rate diagnostic lines. Models with $\beta=2.0$ (blue short dashed line) and $\beta=0.8$ (red long dashed line) are compared to the observed profiles of star 2 (black solid line). Models are for $T_{\text {eff }}=40000 \mathrm{~K}, \dot{M}=$ $10^{-8.5} M_{\odot} \mathrm{yr}^{-1}$ and $V \sin i=300 \mathrm{~km} \mathrm{~s}^{-1}$. The $\mathrm{C}$ IV $\lambda \lambda 1548,1551$ line is almost unchanged while the $\mathrm{N} v \lambda 1238,1242$ line shows narrower absorption and emission when $\beta$ is higher.

the dependence of the size of the interaction region can be seen in Fig. 8.6 of Lamers \& Cassinelli (1999).

In our case, low values of $\beta$ seem to be preferred as the observed $\mathrm{N}$ V $\lambda 1238,1242$ profile does not show a double absorption line. However, $\beta=2.0$ reproduces better the blue edge of the absorption profile. As $\beta=0.8$ is closer to the predictions of the radiation driven wind theory, we adopt this value as typical of the SMC-N81 stars. But whatever the exact value of $\beta$, the mass loss rate determination is not strongly modified as the level of emission remains roughly the same (see Fig. 6).

\section{- Clumping effects:}

As already mentioned, there are indications that the winds of O stars are clumped (Crowther et al. 2002; Hillier et al. 2003; Bouret et al. 2003; Repolust et al. 2004), although there exist no quantitative constraints. The effects of inhomogeneous winds are twofold: first, due to the presence of overdensities, the emission of density-sensitive lines is strengthened; second, the higher density in clumps increases the recombination so that the ionisation is reduced. The competition between the two effects can lead to either stronger or weaker emission. To investigate deeper the effect of clumping on the wind profiles, we have run test models with $f_{\infty}=0.01$ (recall that the filling factor $f$ is given by $\left.f=f_{\infty}+\left(1-f_{\infty}\right) \mathrm{e}^{\frac{-v}{v_{\text {init }}}}\right)$. The main effects on the $\mathrm{N} V \lambda 1238,1242$ and $\mathrm{C}$ IV $\lambda \lambda 1548,1551$ lines are shown

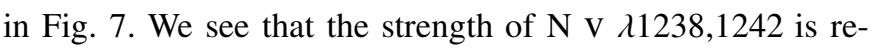
duced and that the $\mathrm{C}$ IV $\lambda \lambda 1548,1551$ absorption is increased in the outer part of the atmosphere. This is attributed to a reduced ionisation in the outer wind ( $\mathrm{N}$ V recombines to $\mathrm{N}$ IV and $\mathrm{C} \mathrm{V}$ to $\mathrm{C}$ IV), which improves the fits. Hence, there is no doubt that 

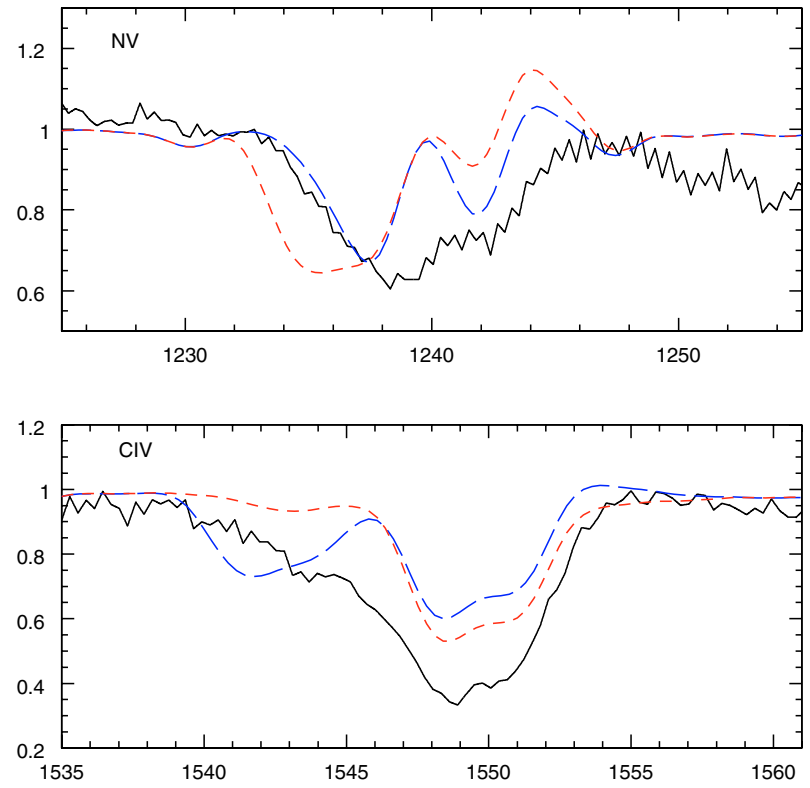

Fig. 7. Influence of clumping on the mass loss rate diagnostic lines. Models with a volume filling factor of 0.01 (blue long dashed line) and 1.0 (red short dashed line, no clumping) are compared to the observed profiles of star 2 (black solid line). Models are for $T_{\text {eff }}=40000 \mathrm{~K}$ and $\dot{M}=10^{-8.5} M_{\odot} \mathrm{yr}^{-1}$ and $V \sin i=300 \mathrm{~km} \mathrm{~s}^{-1}$. The strength of $\mathrm{N} \mathrm{V} \lambda 1238,1242$ is reduced and the absorption of C IV $\lambda \lambda 1548,1551$ is increased when clumping is included.

the inclusion of clumping is crucial to reproduce the observed features. Whether this is a proof of the inhomogeneity of $\mathrm{O}$ star winds or just a trick to simulate the correct ionisation is not clear, but this parameter turns out to be an important ingredient of the modeling. As regards the mass loss determination, Fig. 7 shows that the emission is slightly reduced when clumping is included so that the upper limit on $\dot{M}$ must be slightly increased.

\section{- Adiabatic cooling:}

In low density winds, due to the reduction of any cooling processes based on atomic mechanisms, adiabatic cooling is expected to be important to set the temperature structure (e.g., Drew 1985). Figure 8 demonstrates that this is indeed the case: a model with adiabatic cooling (long dashed line) shows a strong drop in temperature in the outer wind. However, as in this part of the atmosphere populations are mostly governed by radiative processes, the influence on the line profiles is reduced: the C IV $\lambda \lambda 1548,1551$ line shows a slightly enhanced absorption which improves the fit but remains marginal. We conclude that adiabatic cooling is not a crucial parameter as regards the fit of $U V$ wind lines.

\section{- Abundances:}

Metallicity in our models has been chosen to be $1 / 8$ solar, and the individual abundances have simply been scaled according to this global metallicity. A better assumption would have been to take abundances typical of the SMC molecular clouds since the N81 stars are young and their atmospheres probably not contaminated by stellar nucleosynthesis products. Such initial
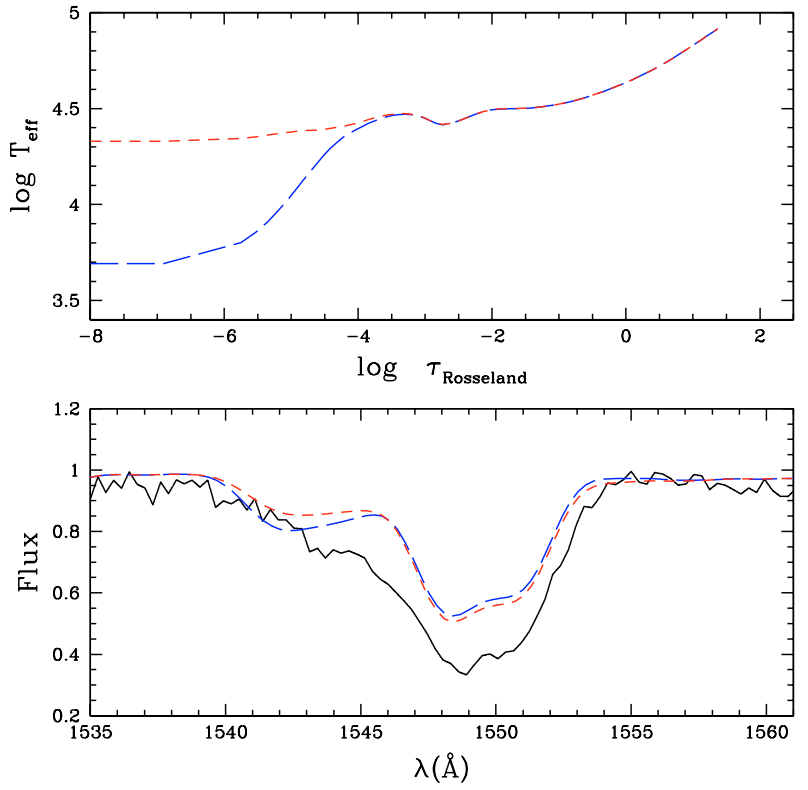

Fig. 8. Effect of the inclusion of adiabatic cooling in model computations on the temperature structure (top) and the C IV $\lambda \lambda 1548,1551$ profile (bottom; blue long dashed line: model with adiabatic cooling; red short dashed line: model without adiabatic cooling). While the temperature is strongly reduced in the outer parts, the C IV $\lambda \lambda 1548,1551$ profile is only slightly affected. Other wind lines do not show any change.

abundances can be obtained by studies of either main sequence stars which have not yet experienced internal mixing, or HII regions. However, few of the former have been undertaken and they are often uncertain (Venn 1999, and references therein), and studies of nebular abundances can lead to underestimates due to the depletion of material on dust grains. Nonetheless, reasonable agreement between these two types of determinations can sometimes be obtained (see Venn 1999, for a discussion) and point to the following values: $\mathrm{C} / \mathrm{C}_{\odot}=1 / 10$, $\mathrm{N} / \mathrm{N}_{\odot}=1 / 20$ and $\mathrm{O} / \mathrm{O}_{\odot}=1 / 5($ Venn 1999; Heap 2003; Vermeij 2002). Adopting these abundances in our computations leads to weaker $\mathrm{N}$ V $\lambda 1238,1242$ and $\mathrm{C}$ IV $\lambda \lambda 1548,1551$ profiles for a given $\dot{M}$. This means that our upper limit on the mass loss rate necessary to remove emission in the wind lines has to be increased. Recently, Asplund (2003) has revised the solar $N$ abundance. With his new value, the SMC-N81 $N$ abundance derived by Vermeij et al. (2002) is 1/30 solar, meaning that the upper limits on $\dot{M}$ have again to be increased a little.

From the above discussion the result is that if the $\mathrm{CNO}$ abundances are reduced and set to more realistic values and if clumping is included, the upper limit on $\dot{M}$ has to be increased by $\sim 1.0$ dex (the effect of abundances being dominant). Clumping also helps to get better shapes of the wind lines. $\beta$ and adiabatic cooling have almost no influence on the $\dot{M}$ determination. As a consequence, we adopt an upper limit on the mass loss rate of star 2 of $10^{-8.0} M_{\odot} \mathrm{yr}^{-1}$.

\subsection{Reliability of the mass loss rate determination}

In view of the low values of $\dot{M}$ derived, we may wonder whether our determination is not hampered by any modeling 
problem. In particular, what is really determined through fits of UV wind lines is the product of the mass loss rate times the ionisation fraction $\left(q_{\mathrm{i}}\right)$ of the absorbing/emitting ion. Any problem with the prediction of these ionisation fractions would translate into an error in $\dot{M}$. To investigate this point, two kinds of tests have been pursued.

\section{- $\mathrm{H}_{\alpha}$ vs. UV mass loss determination:}

$\mathrm{H}_{\alpha}$ is much less sensitive to the model predictions concerning the ionisation than UV resonance lines, so that we have compared the values of $\dot{M}$ derived from the fit of UV lines on the one hand and $\mathrm{H}_{\alpha}$ on the other hand. To this aim we have chosen as a test case the star HD $217086(\mathrm{O} 7 \mathrm{Vn})$ for which constraints on the mass loss rate (Puls et al. 1996; Repolust et al. 2004) and the ionisation fractions of several ions (Lamers et al. 1999) exist. The low density of the wind (see Lamers et al. 1999) and the effective temperature of the order of $37000 \mathrm{~K}$ make this star similar to the SMC-N81 stars, although the wind is less weak. We have computed various models to fit simultaneously the $\mathrm{H}_{\alpha}$ profile and the UV resonance lines.

Figure 9 shows the results of the fits of the wind-sensitive lines. Two types of conclusions can be drawn depending on the value of the $\beta$ parameter (slope of the wind velocity field):

- if we adopt $\beta=0.8$ as derived by Repolust et al. (2004), the $\mathrm{H}_{\alpha}$ profile is best fitted for $\dot{M}=10^{-6.4} M_{\odot} \mathrm{yr}^{-1}$ (dot-dashed line) but in that case the UV lines (especially N IV 11718 ) are too strong. A value of $\dot{M}$ of $10^{-7.2} M_{\odot} \mathrm{yr}^{-1}$ (long dashed line) has to be adopted to improve the fit of these UV lines, but now the $\mathrm{H}_{\alpha}$ absorption is too strong. Mass loss rates derived from the UV seem then to be lower by a factor 6 compared to the $\mathrm{H}_{\alpha}$ determination. Note that the predictions of Vink et al. (2001) give $\dot{M}=10^{-6.1} M_{\odot} \mathrm{yr}^{-1}$ for HD 217086;

- when we increase $\beta$ to 1.7 , a mass loss rate of $10^{-7.0} M_{\odot} \mathrm{yr}^{-1}$ gives a reasonable - although not perfect - agreement between observations and models for both $\mathrm{H}_{\alpha}$ and the UV lines (dotted line in Fig. 9).

While the conclusions of the case $\beta=0.8$ point to a problem with the ionisation fractions predicted by the CMFGEN models, the case $\beta=1.7$ indicates that this problem partly disappears when $\beta$ is increased. This does not necessarily mean that $\beta=1.7$ (the determination of this parameter in low-density winds is difficult, see e.g., Puls et al. 1996), but it is a way to produce the ionisation throughout the wind leading to an overall good fit of the observed profile ${ }^{2}$. Practically, as we do not have any constraint on $\beta$ (see Sect. 5.2.2) we can conclude that in the worst case $\dot{M}$ derived from $U V$ lines is underestimated by a factor 6 , at least if we take the mass loss rate from $\mathrm{H}_{\alpha}$ as the correct one. In fact, if we take the case $\beta=1.7$ as representative of the real conditions in the wind, the error we make when we only look at the UV spectrum (as for our SMC-N81 stars) and adopt $\beta=0.8$ is $0.2 \mathrm{dex}$, or less than a

\footnotetext{
${ }^{2}$ As mentioned in Sect. 5.2.2 clumping can also modify the ionisation in the wind. However, several tests have shown that it was not possible to fit simultaneously $\mathrm{H}_{\alpha}$ and UV lines with clumped models.
}
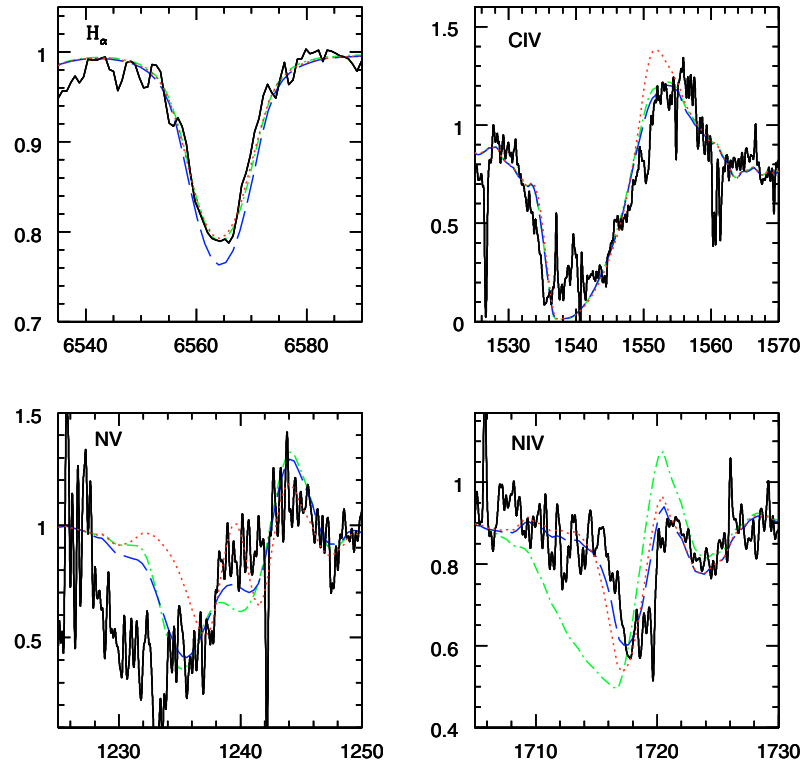

Fig. 9. $\mathrm{H}_{\alpha}$ versus UV lines $\dot{M}$ determinations. The solid line is the observed profile of wind sensitive lines of HD 217086. Other lines are CMFGEN models at $37000 \mathrm{~K}$ with $\beta=0.8$ and $\dot{M}=10^{-6.4} M_{\odot} \mathrm{yr}^{-1}$ (dot-dashed line), $\beta=0.8$ and $\dot{M}=10^{-7.2} M_{\odot} \mathrm{yr}^{-1}$ (long dashed line) and $\beta=1.7$ and $\dot{M}=10^{-7.0} M_{\odot} \mathrm{yr}^{-1}$ (dotted line). A rotational velocity of $290 \mathrm{~km} \mathrm{~s}^{-1}$ has been adopted. For $\beta=0.8$ values of $\dot{M}$ different by one order of magnitudes are required to fit $\mathrm{H}_{\alpha}$ on the one hand and the UV lines on the other hand, while for $\beta=1.7$ a reasonable fit of all lines is achieved. See text for discussion.

factor $2^{3}$. Moreover, in their recent study of SMC dwarfs with weak winds, Bouret et al. (2003) have been able to fit simultaneously lines of different ionisation stages of the same element with CMFGEN models, which indicates that the wind ionisation was predicted correctly.

\section{- SEI method:}

We have applied the SEI method (Lamers et al. 1987) to our SMC N81 star 2. This method leads to the determination of $\dot{M} \times q_{\mathrm{i}}$. Basically, the SEI method solves the radiative transfer in an expanding atmosphere for which the source functions are calculated in the Sobolev approximation. The main input parameters are the velocity field and a function giving the optical depth of the line as a function of velocity. The main output is the value of $\dot{M} \times q_{\mathrm{i}}$. The best fit to the C IV $\lambda \lambda 1548,1551$ line of star 2 is shown in Fig. 10. The corresponding value of $\log \left(\dot{M} \times q_{\mathrm{i}}\right)$ is -9.68 . We also show in this figure the influence of the underlying photospheric absorption: as expected, the low-velocity part of the C IV $\lambda \lambda 1548,1551$ absorption profile is sensitive to this photospheric absorption. As the interstellar contamination renders the true stellar absorption uncertain (see above), we do not give too much weight to this part of the profile which can always be fitted by tuning the photospheric absorption. The important point is that the wind profile $\left(0.4 \leq \Delta v / v_{\infty} \leq 1.0\right)$ is insensitive to the amount of photospheric absorption and only depends on the wind parameters.

\footnotetext{
3 Compared to the value given by Repolust et al. (2004) $-\dot{M}=$ $10^{-6.64} M_{\odot} \mathrm{yr}^{-1}-$, our best determination $\left(\dot{M}=10^{-7.0} M_{\odot} \mathrm{yr}^{-1}\right.$, $\beta=1.7$ ) is a factor $\sim 2$ lower.
} 


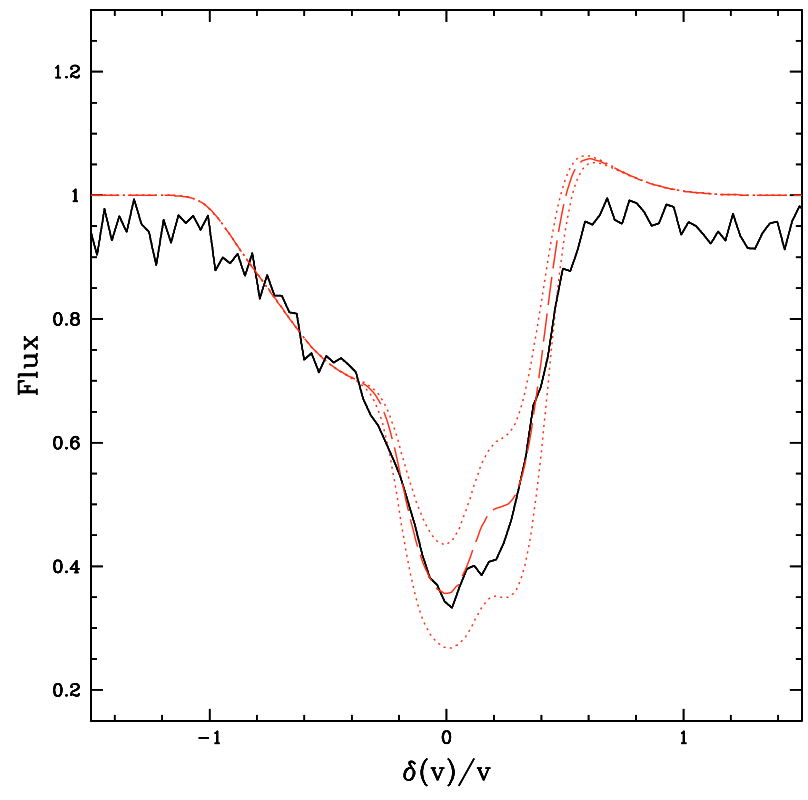

Fig. 10. Fit of C IV $\lambda \lambda 1548,1551$ line with the SEI method. The solid line is the observed profile and the dashed line is the best fit. The parameters for the velocity law are $\beta=1.0$ and $v_{\infty}=1800 \mathrm{~km} \mathrm{~s}^{-1}$. A photospheric component has been used. The optical depth has the following velocity dependence: $\tau(w)=T_{\text {tot }}\left(1-(w)^{1 / \beta}\right)(w)^{0.2}$ with $w=v / v_{\infty}$ and $T_{\text {tot }}=\int_{0.01}^{1} \tau(w)$. The derived value of $\dot{M} \times q_{\mathrm{i}}$ is $10^{-9.68}$. Dotted lines show the influence of an increased or reduced photospheric component: the wind part of the absorption is not affected by this component.

Our estimation of $\dot{M} \times q_{\text {i }}$ with the SEI method can then be regarded as reliable.

To compare this value to that deduced from our model giving the best fit for star 2, we have computed the ionisation fraction of C IV using the definition of Lamers et al. (1999):

$q_{\mathrm{i}}=\frac{\int_{x_{0}}^{x_{1}} n_{\mathrm{i}}(x) \mathrm{d} x}{\int_{x_{0}}^{x_{1}} n_{\mathrm{E}}(x) \mathrm{d} x}$

where $n_{\mathrm{i}}$ is the population of the absorbing ion, $n_{\mathrm{E}}$ the population of the element, $x_{0}\left(x_{1}\right)$ the lower (higher) integration limit ( $x$ being $r / R_{\star}$ ). We found $\log q_{\mathrm{C} \text { IV }}=-1.36$ which, together with a mass loss rate of $10^{-8.5} M_{\odot} \mathrm{yr}^{-1}$ gives $\log \left(\dot{M} q_{\mathrm{i}}\right)=$ -9.86 , in reasonable agreement with the SEI result. This shows that the value of $\dot{M} \times q_{\text {i }}$ obtained by fitting the CMFGEN model profiles to the observations is correct. Thus it follows that if $\dot{M}$ is underestimated, the C IV ionisation fraction is overestimated. To investigate this point, we have compared our ionisation fractions to those derived by Lamers et al. (1999). For stars of different stellar and wind properties, they found a mean $q_{\mathrm{CIV}}$ of the order $10^{-2.5}$ which is $\sim$ a factor of 10 lower than the CMFGEN value. An error by such a factor in $q_{\text {i }}$ translates to an underestimate of $\dot{M}$ by the same factor. This confirms the result of the previous section where a possible underestimate of $\dot{M}$ by a factor 6 was highlighted.

An interesting comment to make is that if we simply assume that the derived mass loss rate is correct, the mean density in the wind (as defined by Lamers et al. 1999) is of the order $10^{-16 \ldots-17} \mathrm{~g} \mathrm{~cm}^{-3}$ which is well below the lowest density probed by the Lamers et al. sample (see their Fig. 3). As their analysis indicates an increase of the $\mathrm{C}$ IV ionisation fraction with decreasing mean density, it is conceivable that for very weak winds $q_{\text {C IV }}$ may reach values of the order of 0.1 , which would reconcile the CMFGEN and Lamers et al. ionisation fractions. Moreover, it should be noted that the study of Lamers et al. includes radio and $\mathrm{H}_{\alpha}$ mass loss rates which did not take clumping into account. Since the inclusion of clumping leads to lower $\dot{M}$ (e.g., Hillier et al. 2003), it is conceivable that some of the Lamers et al. ionisation fractions are underestimated.

The main conclusion of these two types of studies $\left(\mathrm{H}_{\alpha} / \mathrm{UV}\right.$ and SEI) is that there are indications that the ionisation fractions predicted by CMFGEN may be wrong, but by no more than a factor of $\sim 10$ (leading in that case to an underestimation of $\dot{M}$ by the same factor). However, in both studies we have also found means to explain the observations with the CMFGEN predictions (changing the value of $\beta$ in the study of HD 217086, extrapolating the trend $q_{\mathrm{C} \text { IV }}-$ mean density in the SEI study). It is thus not clear if the ionisation fraction predictions of the CMFGEN models are erroneous or not, especially given that we are in a range of parameters never explored before (weak winds). However, if these predictions are wrong, they do not qualitatively modify the conclusion concerning the weakness of the winds (see Sect. 7).

\subsection{Terminal velocity}

The terminal velocity of the winds of $\mathrm{O}$ stars is usually derived from the blueward extension of UV resonance lines. Here, due to the weakness of the outer wind density, the absorption may not extend up to $v_{\infty}$ so that with the above method one can only derive lower limits for the terminal velocities. As shown in Table 4, these limits can even be lower than the escape velocity (which is of the order $1100 \mathrm{~km} \mathrm{~s}^{-1}$ ), reinforcing the fact that the absorption probably does not extend up to $v_{\infty}$. Moreover, the relatively low signal to noise ratio of our spectra coupled to the uncertainty in the flux normalisation introduces an uncertainty in the exact position of the most blue-shifted absorption. Figure 11 shows the $\mathrm{N} v \lambda 1238,1242$ and $\mathrm{C}$ IV $\lambda \lambda 1548,1551$ profiles of models with $v_{\infty}=1500$ and $1800 \mathrm{~km} \mathrm{~s}^{-1}$ compared to the observed line. The $\mathrm{N} v \lambda 1238,1242$ profile is not affected by the change of $v_{\infty}$ while the C IV $\lambda \lambda 1548,1551$ profile is hardly modified. In view of this result, and as the fit with the SEI method requires a value of $1800 \mathrm{~km} \mathrm{~s}^{-1}$ for the terminal velocity, we adopt this value as representative for star 2 . The values for the other stars are given in Table 4.

\subsection{Turbulent velocity/rotational velocity}

The turbulent velocity in $\mathrm{O}$ stars is thought to increase from values of a few $\mathrm{km} \mathrm{s}^{-1}$ near the photosphere to $\sim 10 \%$ of the terminal velocity in the outer atmosphere. As mentioned in Sect. 5.1, iron lines are sensitive to $v_{\text {turb }}$ so that once $T_{\text {eff }}$ is known, they can be used to determine the turbulent velocity. Figure 12 shows the comparison of iron lines of a model with $T_{\text {eff }}=40000 \mathrm{~K}$ but different $v_{\text {turb }}$. For each model, spectra convolved with rotational velocities of 200,250 and $300 \mathrm{~km} \mathrm{~s}^{-1}$ 

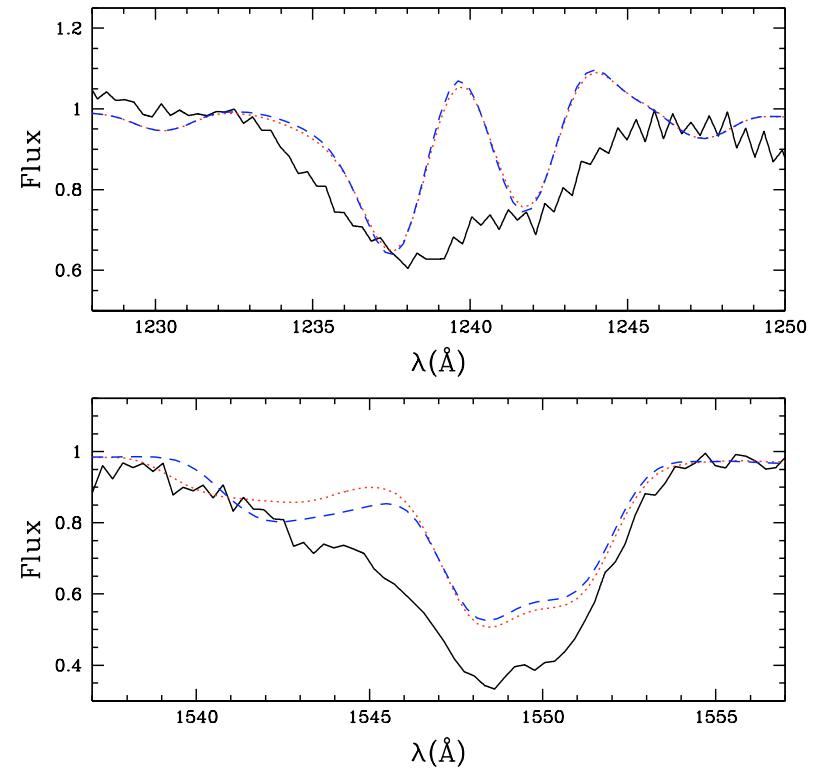

Fig. 11. Determination of $v_{\infty}$. The solid line is the observed spectrum while the dotted (dashed) line is a model with $v_{\infty}=$ 1800 (1500) $\mathrm{km} \mathrm{s}^{-1}$. No change is seen in $\mathrm{N} \mathrm{V} \mathrm{\lambda 1238,1242,}$ and C IV $\lambda \lambda 1548,1551$ is hardly affected. See text for discussion.

are shown. The best fit is obtained for a turbulent velocity of $5 \mathrm{~km} \mathrm{~s}^{-1}$. For higher values, the Fe $\mathrm{V}$ lines deepen too much compared to the observed spectrum. The Fe IV lines behave similarly but also weakly. Tests have been run with a turbulent velocity varying from $5 \mathrm{~km} \mathrm{~s}^{-1}$ near the photosphere up to $100 \mathrm{~km} \mathrm{~s}^{-1}$ in the outer wind and have revealed very little change in the blue part of wind profiles. This is explained by the weakness of the wind in which absorption is likely to take place up to velocities lower than the terminal velocity of the wind. Figure 12 also reveals that the rotational velocity of the star is of the order $300 \mathrm{~km} \mathrm{~s}^{-1}$. Indeed, lower velocities lead to narrow and deep profiles which are not observed.

\subsection{Luminosity}

The luminosity of the stars has been estimated from the absolute visual magnitude $M_{\mathrm{V}}$ and a bolometric correction calculated for the estimated $T_{\text {eff }}$ of the star according to

$\log \frac{L}{L_{\odot}}=-0.4\left(M_{\mathrm{V}}+B C-M_{\odot}^{\mathrm{bol}}\right)$

where $M_{\odot}^{\text {bol }}=4.75$ (Allen 1976). $M_{V}$ was derived in Paper I from the observed visual magnitude and the estimated extinction. $B C$, which is essentially model-independent when calculated as a function of $T_{\text {eff }}$, is derived from the relation of Vacca et al. (1996)

$B C\left(T_{\text {eff }}\right)=27.66-6.84 \times \log T_{\text {eff }}$.

The uncertainty is 0.01 for the observed visual magnitude (Paper I), 0.05 for the extinction (from an uncertainty of 0.01 on $E(B-V)$ ), 0.025 for the distance modulus (di Benedetto 1997) and finally 0.25 for $B C$ for a typical error on $T_{\text {eff }}$ of $3000 \mathrm{~K}$. On average, the uncertainty in $L$ is therefore of the order of 0.12 dex.
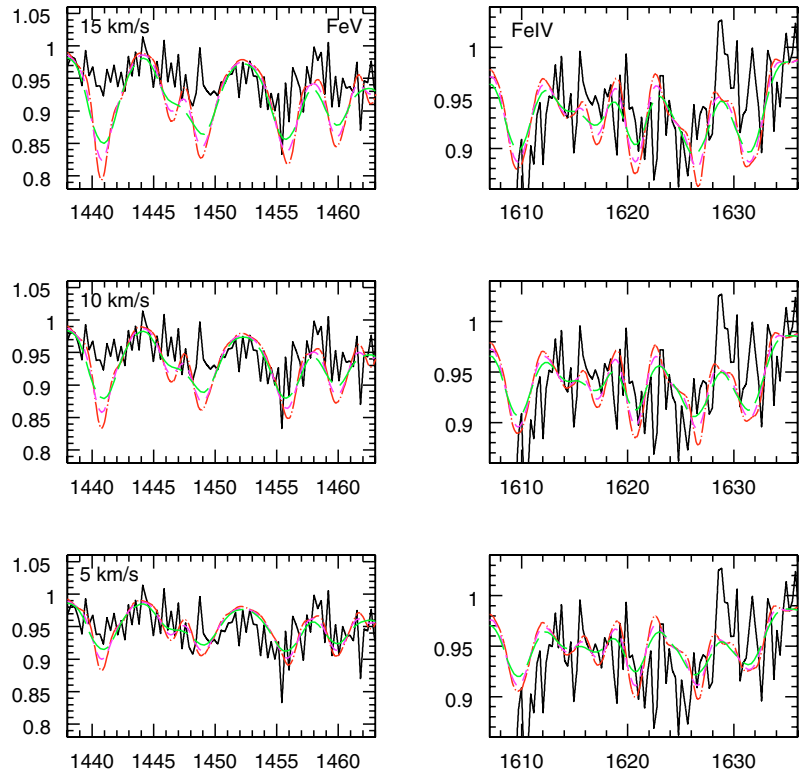

Fig. 12. Effect of microturbulence and rotation on the iron spectrum. The three left panels show Fe V lines while the Fe IV lines are shown in the right panels. The turbulent velocity decreases from 15 to $5 \mathrm{~km} \mathrm{~s}^{-1}$ from top to bottom. Each model is convolved with a rotational velocity of 100 (dot - long dashed line), 200 (short dashed line) and $300 \mathrm{~km} \mathrm{~s}^{-1}$ (long dashed line). The lower panels $\left(v_{\text {turb }}=5 \mathrm{~km} \mathrm{~s}^{-1}\right)$ give the best fit.

\subsection{Summary and results for other stars}

Stellar and wind parameters for 3 other stars of SMC-N81 have been determined with a similar analysis. Here we summarise the results of this analysis and give the results in Table 4. The best fits are shown in the Appendix. For the remaining stars presented in Paper I, no constraints have been derived due to the poor quality of the spectra. For all the stars, $\beta=0.8$ and $\mathrm{Si}$, $\mathrm{S}$ and $\mathrm{Fe}$ abundances equal to $1 / 8$ solar have been chosen. The masses have been derived from the HR diagram presented in Fig. 14.

\subsubsection{Star 2}

The main results have been given in the previous sections. In contrast to all other stars, we stress that the fits of the C IV $\lambda \lambda 1548,1551$ and $\mathrm{N}$ V $\lambda 1238,1242$ lines for star 2 are improved when the abundances are taken to be $1 / 8$ solar. Choosing the lower values indicated by the nebular analysis (see Sect. 5.2.2) leads to too weak absorptions even when clumping is included. The best fits are achieved with a filling factor at the top of the atmosphere of $0.01^{4}$.

\subsubsection{Star 1}

For this star, $T_{\text {eff }}$ is found to be $\sim 38500 \mathrm{~K}$. The upper limit on the mass loss rate is $10^{-8.0} M_{\odot} \mathrm{yr}^{-1}$ and $v_{\infty}$ is at least $1500 \mathrm{~km} \mathrm{~s}^{-1}$. Clumping is necessary to improve the fit

\footnotetext{
${ }^{4}$ Recall that in the present parameterisation of clumping in CMFGEN, this means that $f$ goes from 1 at the photosphere to 0.01 when $v=v_{\infty}$.
} 
of the C IV $\lambda \lambda 1548,1551$ line $\left(f_{\infty}=0.01\right)$. A reasonable fit is achieved with the nebular $\mathrm{CNO}$ abundances.

\subsubsection{Star 3}

Star 3 is the coolest of the 4 stars studied $\left(T_{\text {eff }}=36000 \mathrm{~K}\right)$. The determination of the mass loss rate is very difficult because the $\mathrm{N}$ V $\lambda 1238,1242$ line is almost absent due to the low $T_{\text {eff }}$ and the C IV $\lambda \lambda 1548,1551$ line is almost entirely photospheric. Hence, secondary indicators such as O IV $\lambda \lambda 1339,1343$ have been used to estimate $\dot{M}$ (which is found to be lower than $10^{-8.5} M_{\odot} \mathrm{yr}^{-1}$ ). We want to stress that this determination is probably the most uncertain of our sample, especially since the the observed spectrum is noisy. The very small value for the terminal velocity reflects the low density of the wind: the number of absorbants in the wind is so low that the wind lines are essentially absent. The nebular $\mathrm{CNO}$ abundances give the best fits.

\subsubsection{Star 11}

We have estimated an effective temperature of $37000 \mathrm{~K}$ for this star. An upper limit of $10^{-9.0} M_{\odot} \mathrm{yr}^{-1}$ is estimated from the $\mathrm{N} V \lambda 1238,1242$ and $\mathrm{C}$ IV $\lambda \lambda 1548,1551$ lines. The terminal velocity we derive $\left(600 \mathrm{~km} \mathrm{~s}^{-1}\right)$ is again a lower limit. The nebular $\mathrm{CNO}$ abundances give reasonable fits and clumping is not necessary.

\section{Nebular and stellar properties}

In this section we first go back to the nebular properties of N81 and then investigate the evolutionary status of the individual stars together with the consequences of their weak winds.

\subsection{Nebular properties/lonising fluxes}

A meaningful way of testing our results concerning the stellar properties of the N81 individual components is to compare them to the integrated properties of the cluster.

First, Heydari-Malayeri et al. (1999) derived a mean extinction of $A_{\mathrm{V}}=0.40$ from the observed $\mathrm{H}_{\alpha} / \mathrm{H}_{\beta}$ ratio in the nebula, and they used this value to correct the observed flux in $\mathrm{H}_{\beta}$ and to estimate the number of Lyman continuum photons $-Q_{0}$ - emitted by the N81 stars (under the assumption that the HII region is ionisation bounded). They find $Q_{0}=$ $1.36 \times 10^{49}$ photons $^{-1}$. From this and from the calibration of Vacca et al. (1996), they conclude that a single main sequence star of spectral type 06.5 or 07 can lead to such an ionising flux. We have estimated the total amount of ionising photons released by the N81 stars from the SEDs of the models giving the best UV fit. As the N81 stars studied here are the most luminous and hottest of the region, they are likely to provide essentially all the ionising flux. We found from the models $Q_{0}=1.64 \times 10^{49}$ photons s $^{-1}$ in good agreement with the values derived from the nebular properties. The lower value of the estimate of Heydari-Malayeri et al. (1999) can be partly explained by the fact that the HII region may be density bounded so that a part of the ionising flux may escape the cavity.
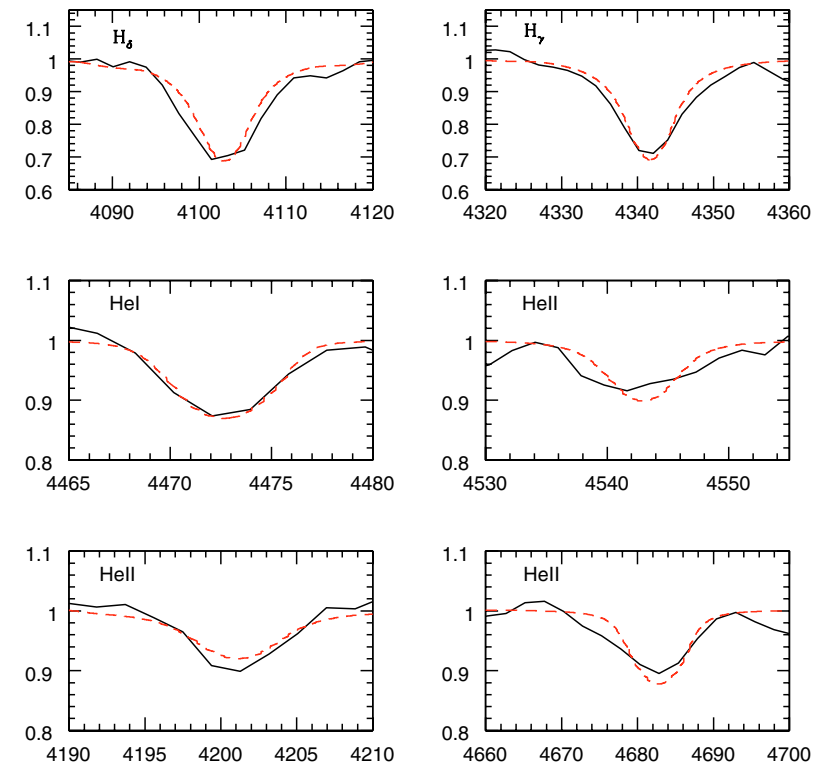

Fig. 13. Comparison between observed optical spectrum of the N81 cluster corrected for nebular lines (solid line) and sum of the optical spectra of the models giving the best UV fits (dashed line). Each optical spectrum from the models has been convolved with rotational velocities derived from the UV spectra. The agreement is very good, showing that our determination of the stellar parameters of the N81 stars is reliable.

Second, we have integrated optical spectra of the N81 cluster that have been corrected for the nebular contamination so that they give the total stellar spectrum which can be compared to the sum of the individual stellar optical spectra of the models giving the best UV fits. Figure 13 shows that there is again a good agreement between the observed and modeled spectra. From the strength of the He lines, a "mean" spectral type $\mathrm{O} 7$ is found (which is in fact similar to the spectral type of the two most luminous stars of the cluster, namely stars 1 and 2). This indicates that despite the difficulties in estimating the stellar properties of the individual stars (in particular $T_{\text {eff }}$ ), our results are reliable. Moreover, the He II $\lambda 4686$ line is reasonably fitted by the models. As this line is usually filled by wind emission, this may be an indication of not too high mass loss rates.

\subsection{Evolutionary status}

We have placed the N81 stars on an HR diagram constructed with Geneva evolutionary tracks without rotation for $Z=0.004$ (Fig. 14). All the stars are compatible with an age $\leq 5$ Myrs. While star 1 seems to be slightly older, the 3 other stars (especially star 3 and 11) lie close to the ZAMS and have an age between 0 and 4 Myrs. There seems to be an age dispersion of the order 1 or 2 Myrs in the cluster, which is reasonable. The inclusion of rotation is known to move the ZAMS towards lower $T_{\text {eff }}$ (see Meynet \& Maeder 1997), reducing the age estimates for stellar populations compared to the nonrotating case. However, this effect becomes significant only for rotational velocities close to the critical velocity. As the SMC-N81 stars have $V \sin i$ of only $200-300 \mathrm{~km} \mathrm{~s}^{-1}$ (which corresponds to $\sim 1 / 3$ of the break velocity), our results are 


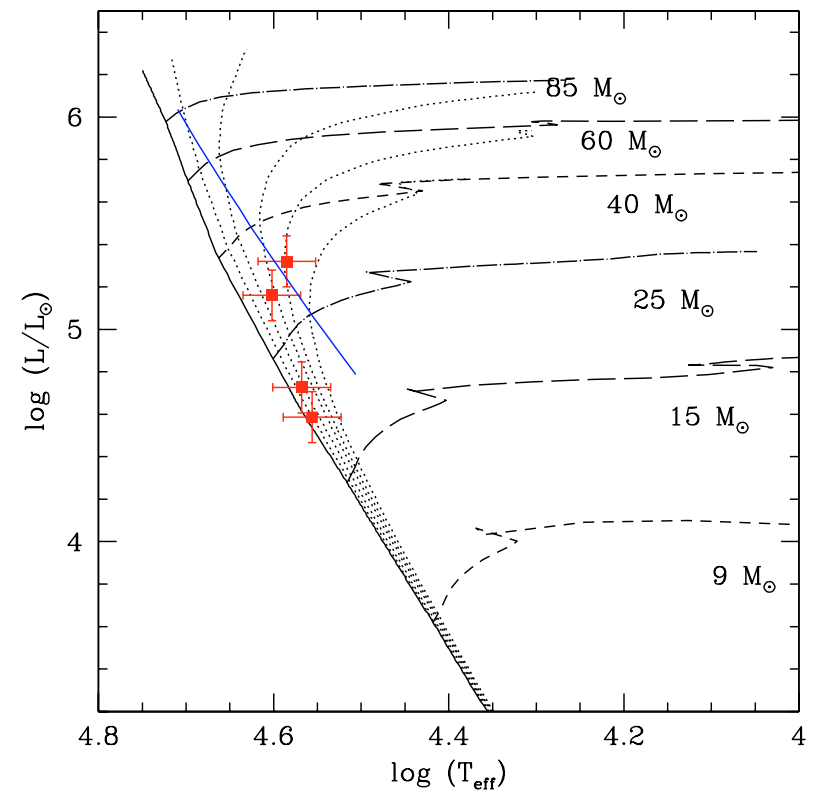

Fig. 14. HR diagram for $Z=0.004$ without rotation. Different Geneva evolutionary tracks for various masses are indicated, together with the ZAMS and isochrones for 1, 2, 3, 4 and 5 Myrs (data from Lejeune \& Schaerer 2001). The filled squares give the position of the SMC-N81 stars with the typical errors on their position. The calibration $\log L-$ $\log T_{\text {eff }}$ of Vacca et al. (1996) for dwarfs is also shown by the long dashed line. Note the underluminosity of most of the SMC-N81 stars.

probably not strongly hampered by the use of stellar tracks without rotation.

Based on the equations governing the dynamical evolution of HII regions (Dyson 1978) with the typical values $\dot{M}=3 \times$ $10^{-9} M_{\odot} \mathrm{yr}^{-1} v_{\infty}=1500 \mathrm{~km} \mathrm{~s}^{-1}, n_{0}=400 \mathrm{~cm}^{-3}$ (gas density, see Heydari-Malayeri et al. 1988) and a radius of the HII region of $3 \mathrm{pc}$, one can derive an age of $\sim 1.4 \mathrm{Myr}$ for the N81 region. This assumes that star 1 and 2 provide most of the mechanical energy $\left(\frac{1}{2} \dot{M} v_{\infty}\right)$. Previous age estimates based on the decrement of $\mathrm{H}_{\beta}$ equivalent width by Heydari-Malayeri et al. (1988) were of 1 to $2.5 \mathrm{Myr}$ assuming a stellar cluster of solar metallicity. However, for clusters with a small number of ionising stars this method is inherently inaccurate due to statistical fluctuations.

If we compare the positions of the SMC-N81 stars with the calibration $L-T_{\text {eff }}$ for dwarfs of Vacca et al. (1996) based on studies of Galactic O stars (long dashed line in Fig. 14), we note that except star 1 , the other components are all underluminous compared to "normal" $\mathrm{O}$ dwarfs. This result based on the quantitative study of the stellar properties confirms the conclusion of Paper I in which the subluminosity of most of the stars was already highlighted and interpreted as an indication of the youth of the stars.

In Paper I it was argued that the SMC-N81 stars could belong to the class of $\mathrm{Vz}$ stars. Figure 14 shows that although young, the stars do not lie perfectly on the ZAMS. This may be an indication that either Vz stars are not strictly ZAMS stars but more probably "young" stars less evolved than "normal" dwarfs, or that our objects are not "true" Vz stars (we recall that we do not dispose of optical spectroscopy to firmly establish if the SMC-N81 stars are Vz stars or not). A deeper investigation of the properties of this interesting class of objects will be presented in a forthcoming paper.

\section{Puzzling wind properties}

\subsection{Comparison to previous observations and theoretical predictions}

One of the most surprising feature of the spectra of the SMC-N81 stars is the shape of the wind lines (mainly $\mathrm{N}$ V $\lambda 1238,1242$ and $\mathrm{C}$ IV $\lambda \lambda 1548,1551)$ : while they show no emission, they display a blueshifted absorption profile. This is quite puzzling: how can we produce such an absorption without any emission? Theoretically, in an extended atmosphere, the absorption in the P-Cygni profile comes from the removal of photons from the observer's line of sight, and the emission is due to photons that have been scattered isotropically on sight lines not parallel to that of the observer. Globally, the amount of "absorbed" photons is then equal to the number of "emitted" photons. If the atmosphere is only weakly extended (i.e. its height is smaller than the stellar radius), then roughly half the photons are backscattered towards the star and destroyed, so that the emission is reduced compared to the absorption (the ratio between them being $1 / 2$ ). This is even more true if there is an underlying photospheric absorption which increases the absorption with respect to the emission. This points to the behaviour observed in our spectra, but the absence of emission remains a puzzle.

A possible explanation for the absence of emission may be an enhanced backscattering of photons towards the photosphere, so that the emission is even more reduced compared to the absorption. But the physical reason for such a process is not known. Another possibility is a strongly non-spherically symmetric wind. Rotation is known to increase the mass loss near the poles and to reduce it at the equator (Bjorkmann \& Cassinelli 1993; Maeder \& Meynet 2000). If a star rotates sufficiently fast to induce a strong contrast between the polar and equatorial ejection, and if we can observe it pole-on, then we can obtain the kind of profile we have: the blueshifted absorption comes from the high density polar flow while the (absence of) emission corresponds to the low density "equatorial" atmosphere. However, as all the SMC-N81 stars show such profiles and as the probability to see all of them poleon is low, this explanation is not satisfying. Moreover, as the projected rotational velocities of the N81 stars are of the order $200 / 300 \mathrm{~km} \mathrm{~s}^{-1}$, it is not likely that they are seen pole-on.

In spite of these curious features, we have been able to place constraints on the wind properties of the SMC-N81 stars, the main results being that they lose mass at an extremely low rate. Whether this is typical or not of $\mathrm{O}$ dwarfs at low metallicity remains to be established. Indeed, this is one of the first quantitative determinations of mass loss rates for such objects and consequently few comparisons are possible. To our knowledge, the only previous studies of wind parameters of $\mathrm{O}$ dwarfs in the SMC have been performed by Puls et al. (1996), Bouret et al. (2003) and very recently Massey et al. (2004). Puls et al. (1996) only derived upper limits on the mass loss rates for four SMC dwarfs. This is mainly due to the fact that their method 


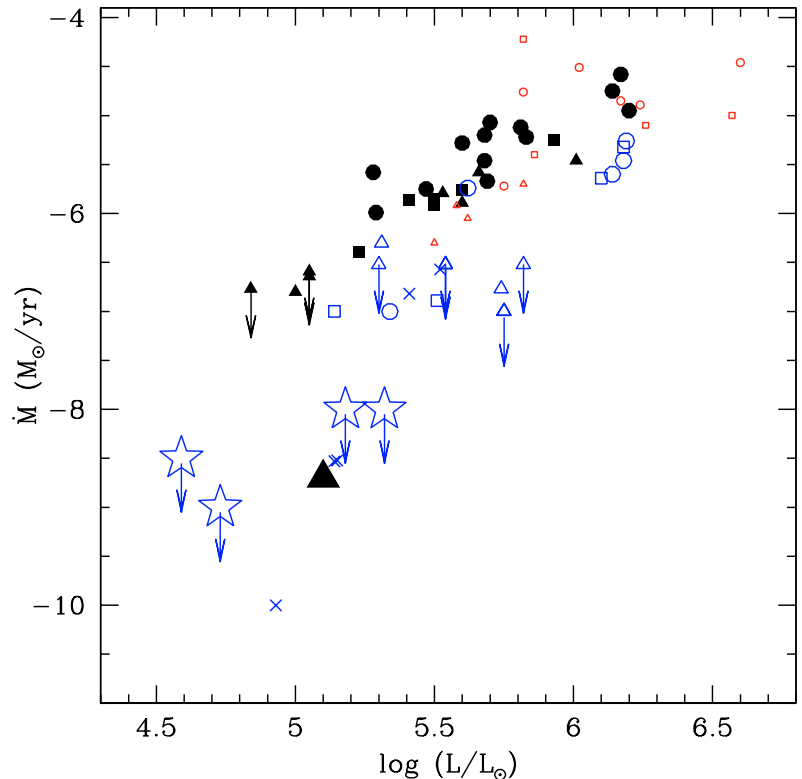

Fig. 15. Mass loss rate as a function of stellar luminosity for $\mathrm{O}$ stars. Filled (open) symbols are Galactic (LMC, small red/SMC, blue) objects. Triangles (squares, circles) are for luminosity class V (III, I). Crosses are the SMC stars of Bouret et al. (2003). Data are from Puls et al. (1996), Herrero et al. (2000), Crowther et al. (2002), Repolust et al. (2004), Hillier et al. (2003) and Massey et al. (2004). The star symbols are for the SMC-N81 stars. Note the low mass loss rates of the SMC objects with $\log \frac{L}{L_{\odot}} \leq 5.5$ and of 10 Lac (large filled triangle).

relies on the the strength of the $\mathrm{H}_{\alpha}$ emission whereas the $\mathrm{H}_{\alpha}$ profile in SMC dwarfs is mostly in absorption. Their upper limits are of the order $10^{-7} M_{\odot} \mathrm{yr}^{-1}$ which is roughly 10 times higher than our estimates for the SMC-N81 stars. The recent work by Bouret et al. (2003) includes 5 SMC O dwarfs of which three (with spectral type between 09.5 and O6) have mass loss rates between $10^{-10}$ and $3 \times 10^{-9} M_{\odot} \mathrm{yr}^{-1}$. Our results agree very well with these estimates (we recall that the SMC-N81 stars have mid to late spectral types). The dwarfs studied by Massey et al. (2004) are more luminous than the N81 stars and have therefore higher mass loss rates. Figure 15 shows mass loss rates as a function of luminosity for Galactic and SMC dwarfs from different studies. The results of this work and of Bouret et al. (2003) indicate a strong reduction of the mass loss rate of stars with $\log \frac{L}{L_{0}} \lesssim 5.5$.

How do these results compare to theoretical calculations? The most recent predictions of wind parameters as a function of metallicity are the extensive calculations by Vink et al. (2001). Using their cooking recipe to estimate the mass loss rate as a function of stellar parameters and metallicity, we found $\dot{M}$ of the order $10^{-7 \ldots 8} M_{\odot} \mathrm{yr}^{-1}$ for the SMC-N81 stars (the exact values are given in Table 4). The derived mass loss rate are more than 30 times lower. Even if we take into account a possible error in the ionisation fraction of the CMFGEN models (which would lead to an increase of the $\dot{M}$ determinations by a factor of $\lessgtr 10)$ the mass loss rates are still lower than predicted (with perhaps the exception of star 3 for which we recall that the $\dot{M}$ determination is uncertain). There is thus no doubt that the winds of the SMC-N81 stars show unusual weakness.

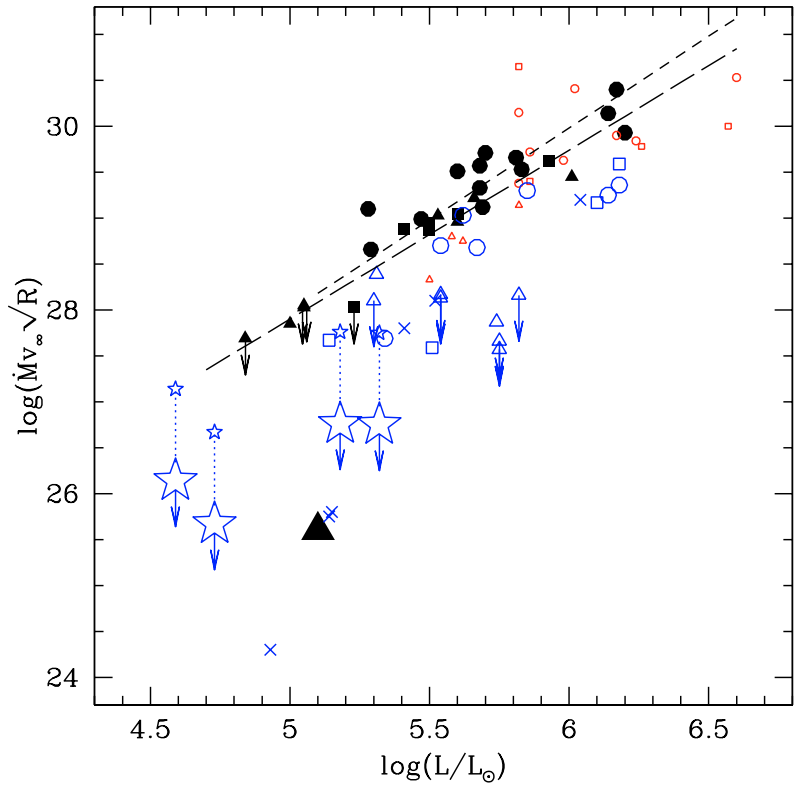

Fig. 16. Modified wind momentum-luminosity relation. Data and symbols are the same as in Fig. 15. The SMC-N81 stars (for which we have adopted $v_{\infty}=2.6 v_{\text {esc }}$ to compute the modified wind momentum) confirm the trend of either a reduced modified wind momentum at low luminosity or a steeper slope of this relation at low metallicity. The small star symbols show the position of the SMC-N81 stars if $\dot{M}$ was systematically underestimated by a factor 10 . The long-dashed (shortdashed) line gives the mean relation for luminosity clas V-III (I) stars from Repolust et al. (2004). The position of $10 \mathrm{Lac}$ is also indicated by the large filled triangle.

This result is even more striking in terms of modified wind momentum. This quantity defined by $\dot{M} v_{\infty} \sqrt{R}$ is predicted to be a power law of the sole stellar luminosity (e.g., Kudritzki \& Puls. 2000) to give the so-called modified wind momentumluminosity relation (hereafter WLR)

$\dot{M} v_{\infty} \sqrt{R} \propto L^{\frac{1}{\alpha}}$.

Figure 16 shows the observed relation for Galactic and MC stars. For Galactic stars, the correlation is well defined and is slightly different for luminosity class I stars and LC class III-V stars (as demonstrated by the regression curves). For the MC objects however, the relation seem to be different from that followed by the Galactic objects, at least for the LC V stars. Indeed, our results indicate that the SMC-N81 stars (star symbols if Fig. 16) have modified wind momenta lower by $\sim 2$ orders of magnitudes compared to Galactic objects of the same luminosity and following the LC III-V relation, which simply reflects the weakness of the winds ${ }^{5}$. Bouret et al. (2003) indicate a similar trend. Note that even if the mass loss rates are underestimated by a factor 10 (see Sect. 5.2.2) there is still a significant difference compared to Galactic objects (except in the case of star 3) as shown by the small star symbols in Fig. 16. We have also compared the derived wind momenta with those expected for B and A

5 To compute the modified wind momenta of the SMC-N81 stars, we have adopted $v_{\infty}=2.6 v_{\text {esc }}$ since we have not been able to derive the true terminal velocities due to the weakness of the winds. 
supergiants (e.g., Kudritzki et al. 1999): it turns out that the SMC-N81 momenta are weaker. This confirms the extreme weakness of the winds of the SMC-N81 stars.

\subsection{Possible origin of the low $\dot{M} /$ wind momentum}

Several possibilities can be invoked to explain the low wind momenta observed for the SMC-N81 stars: first, there may be a breakdown of the modified wind momentum-luminosity relation at low luminosity for dwarfs; second, metallicity can affect this relation; third, ion runaway may happen in the wind, reducing the mass loss rate. In the following, we investigate these different hypotheses. Finally we will speculate on the possible relation between these "unusual" wind properties and the youth of these stars.

1) Breakdown of the wind momentum-luminosity relation at low luminosities:

Let us first consider the case of Galactic stars. The study of Puls et al. (1996) showed that in the luminosity range $5.2<$ $\log L / L_{\odot}<5.5$ several of their stars seemed to indicate a weaker modified wind momentum than expected from the relation followed by higher luminosity stars, indicating a possible break in the slope of the WLR. However, the recent reanalysis of these stars by Repolust et al. (2004) does not show this trend any more if their upper limits are taken as the true values for $\dot{M}$. As their mass loss determinations are based on $\mathrm{H}_{\alpha}$ fitting and as this line becomes insensitive to $\dot{M}$ below $\sim 10^{-8} M_{\odot} \mathrm{yr}^{-1}$ (e.g., Herrero et al. 2002), one cannot rule out the possibility of low wind momenta for these low luminosity objects. UV analysis of these stars could certainly shed more light on this question.

Concerning low metallicity objects, few LMC stars have been studied so far, all of them being furthermore of high luminosity. More SMC stars have been studied (including the SMC-N81 objects). For objects with $\log L / L_{\odot}>5.5$, the wind momenta are lower than for Galactic stars, but the WLR seems to have the same slope. The low luminosity stars show a clear reduction of the wind momenta which imply a steeper slope of the WLR in this luminosity range. Nonetheless, most of the high $L$ objects are giants or supergiants while low $L$ objects are all dwarfs. As the WLR may be different for different luminosity classes, the existence of a real breakdown is unclear.

Moreover, reasons for such a possible breakdown of the wind momentum-luminosity relation are not known. Is it linked with the driving mechanism? One possibility could be a change of the ions responsible for the radiative acceleration with the consequence of the modification of the efficiency of the driving, but this would be linked to the change of the ionisation in the atmosphere and then more to $T_{\text {eff }}$ than to $L$. Is this breakdown of the wind momentum luminosity relation only due to the low luminosities of the stars? There are in fact other objects, central stars of planetary nebulae, which are much less luminous than any $\mathrm{O}$ stars and which seem to follow the mean relation for Galactic objects with $\log \frac{L}{L_{\odot}} \geq 5.5$ (see Kudritzki \& Puls 2000). This renders the behaviour of the low luminosity $\mathrm{O}$ stars even more puzzling.
Another possibility is that the current formalism of the radiation theory may be erroneous in the case of massive stars with weak winds. Indeed, Owocki \& Puls (1999) have shown that in such winds the curvature of the velocity field near the sonic point could lead to an inward directed flux of the diffuse radiation field that can significantly reduce the total radiative acceleration compared to the CAK approach (see their Fig. 7). As a consequence, the mass loss rates are also reduced compared to the predictions based on the CAK formalism for radiative acceleration. For stars with high luminosity and/or low gravity, the density scale height just above the photosphere is higher ${ }^{6}$ so that line thermalisation near the sonic point may suppress the above effect (see Owocki \& Puls 1999). This may explain why giants/supergiants and dwarfs with high luminosity are not sensitive to the effects of the curved velocity field and show a more classical behaviour. This possibility is attractive since it could explain why only dwarfs with low luminosities seem to have wind properties deviating from the predictions of the CAK theory.

Puls et al. (2000) have made a detailed study of the line statistics and its effect on the radiative driving and have shown that under certain conditions, the parameterisation of the radiative acceleration with the CAK formalism $\left(g \propto\left(\frac{\mathrm{d} v}{\mathrm{~d} r}\right)^{\alpha}\right)$ is not valid, and thus the predictions based on this formalism are erroneous. This would happen if both the level density of the ions responsible for the driving were much higher and the distribution of the oscillator strengths were much steeper. However, they argue that the atomic physics of the driving ions should not lead to such extreme conditions.

In summary, the above discussion presents evidence that the current predictions of the radiation driven wind theory may fail to explain the winds of low luminosity $\mathrm{O}$ dwarfs due to subtle effects negligible in the winds of stars studied so far.

\section{2) Metallicity effect:}

The wind momentum-luminosity relation may be different at low metallicity. Such a dependence, though quite weak, is in fact predicted by the radiation driven wind theory. The inverse of the luminosity exponent in Eq. (4) $(\alpha)$ is linked to the line strength distribution function. But this function depends on metallicity, $\alpha$ being lower at low $Z$ (e.g., Abbott 1982; Puls et al. 2000). It turns out that the wind momentum-luminosity relation should have a steeper slope at subsolar metallicities. The reduction of the number of lines effectively driving the wind also leads to a global shift of the relation towards lower modified wind momenta. Our results tend to indicate a steeper slope of the WLR, together with the Puls et al. (1996) SMC objects and the Bouret et al. (2003) results. Nonetheless, the real form of the WLR is

$\dot{M} v_{\infty} \sqrt{R} \propto(M(1-\Gamma))^{\frac{3}{2}-\frac{1}{\alpha}} L^{\frac{1}{\alpha}}$

where $\Gamma$ is the ratio of radiative acceleration to gravitational acceleration. For solar metallicity, $\alpha \sim 2 / 3$ so that the dependence on the effective mass $M(1-\Gamma)$ vanishes. Values

\footnotetext{
${ }^{6}$ The density scale height is proportional to $R_{\star}^{2} / M(1-\Gamma)$ where $R_{\star}$ is the stellar radius and $\Gamma$ the ratio of electron scattering to gravitational acceleration.
} 


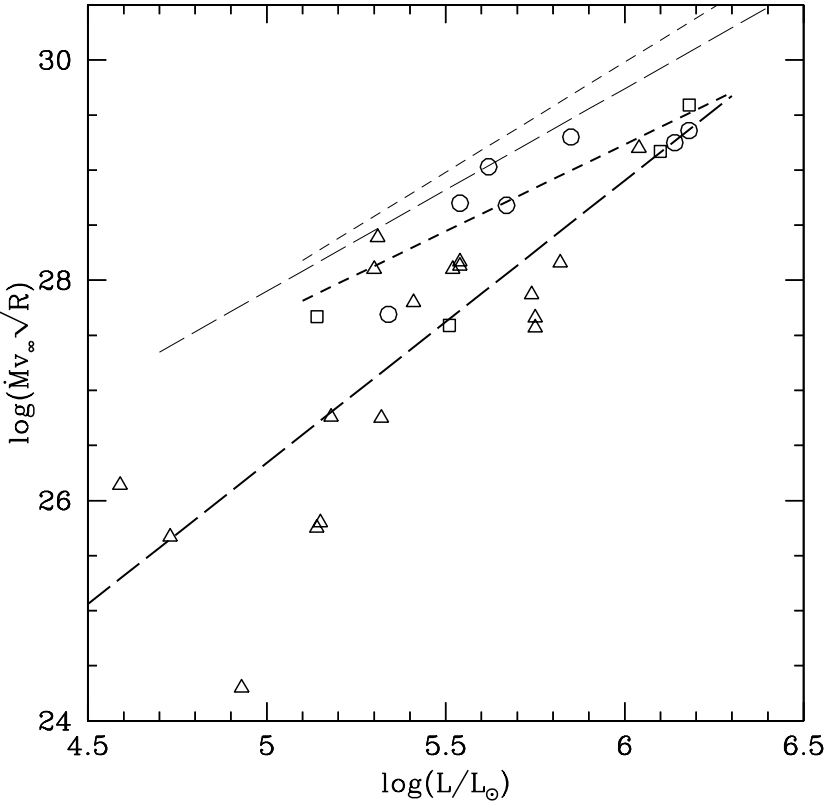

Fig. 17. Modified wind momentum-luminosity relation for SMC stars. Data are from Puls et al. (1996), Bouret et al. (2003), Massey et al. (2004) and the present work. Dwarfs (giants, supergiants) are shown by triangles (squares, circles). The long-dashed (short-dashed) curves are regressions for supergiants (giants/dwarfs) of the Galaxy (light curves, from Repolust et al. 2004) and the SMC (bold curves). SMC giants and dwarfs may indicate a steeper slope of the modified wind momentum-relation at low metallicity, but the scatter is considerable and more studies are needed to confirm this trend.

of $\alpha<2 / 3$ bring back this dependence, which should lead to a large scatter of the WLR for low $Z$ objects and then to a more difficult calibration of this relation. Figure 17 shows the modified wind momentum-luminosity relation for the SMC stars studied so far: the scatter is indeed significant. Indicative regression curves are given and reveal a possibly steeper slope $(\alpha \sim 0.4)$ for giants/dwarfs while for the supergiants $\alpha$ may be close to $2 / 3$. More studies are needed to confirm this trend of a steeper slope at lower metallicities.

However, two points may impede this explanation. First, the most recent hydrodynamical simulations do not show any change of the slope of the WLR at metallicities typical of the Magellanic Clouds (Vink et al. 2001; Hoffmann et al. 2002). Furthermore, Kudritzki (2002) has shown that only for extremely low metal content $-10^{-3} Z_{\odot}$ - a difference appears, although his calculations were made at high luminosities $\left(\log L / L_{\odot}>6\right)$ where most of the driving is thought to be done by $\mathrm{H}$ and $\mathrm{He}$ lines, leading to a weaker metallicity dependence of the wind properties. Second, some Galactic stars are known to display signatures of weaker than normal winds (Walborn et al. 1995) but none has been analysed quantitatively so far.

We have carried out a detailed study of the wind properties of one of them: $10 \mathrm{Lac}$. This O9V star has often been considered as a standard dwarf star for its stellar properties. We have run models to fit its IUE and optical spectra taking the the stellar parameters from Herrero et al. $(2002)^{7}$ and the CNO

\footnotetext{
${ }^{7} T_{\text {eff }}=36000 \mathrm{~K}, \log g=3.95$, and the He abundance $\epsilon=0.09$.
}
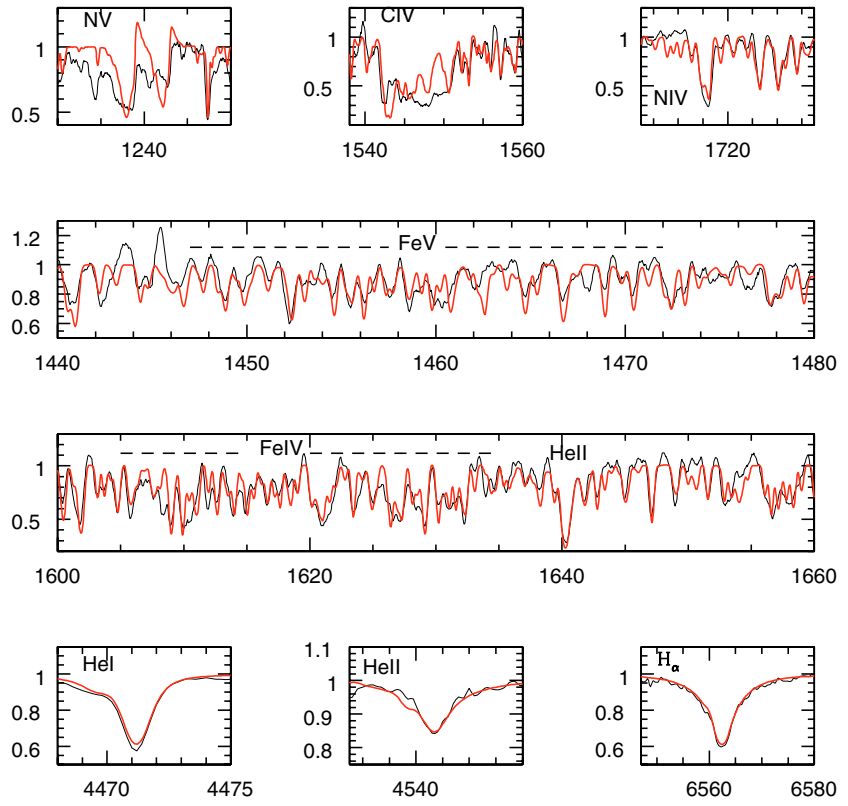

Fig. 18. Fit of the UV and optical spectra of 10 Lac (light curve: observation; bold curve: model). The parameters of the models are: $T_{\text {eff }}=36000 \mathrm{~K}, \log g=4.1, \dot{M}=2 \times 10^{-9} M_{\odot} \mathrm{yr}^{-1} v_{\infty}=1070 \mathrm{~km} \mathrm{~s}^{-1}$. A turbulent velocity increasing from $5 \mathrm{~km} \mathrm{~s}^{-1}$ near the photosphere to $100 \mathrm{~km} \mathrm{~s}^{-1}$ in the outer wind has been adopted. Abundances are from Herrero et al. (2002) for He, Villamariz et al. (2002) for CNO and have been set to the solar value (Grevesse \& Sauval 1998) for the other metals. A rotational velocity of $40 \mathrm{~km} \mathrm{~s}^{-1}$ has been adopted.

abundances from Villamariz et al. (2002). A microturbulent velocity increasing from $5 \mathrm{~km} \mathrm{~s}^{-1}$ near the photosphere up to $100 \mathrm{~km} \mathrm{~s}^{-1}$ in the outer atmosphere was used. Figure 18 shows the results of our best fit model for which $\dot{M}=2 \times$ $10^{-9} M_{\odot} \mathrm{yr}^{-1}$ and $v_{\infty}=1070 \mathrm{~km} \mathrm{~s}^{-1}$. This value of $\dot{M}$ is lower than estimates of Howarth \& Prinja $\left(1989,10^{-6.7} M_{\odot} \mathrm{yr}^{-1}\right.$ based on line profile fitting with a Sobolev code), of Leitherer (1988, $10^{-6.83} M_{\odot} \mathrm{yr}^{-1}$ based on $\mathrm{H}_{\alpha}$ ) and lower than the prediction of Vink et al. (2001) $-10^{-6.2} M_{\odot} \mathrm{yr}^{-1}-$ but it is in agreement with the more recent upper limit of Herrero et al. (2002) based on $\mathrm{H}_{\alpha}$ fits $\left(10^{-8} M_{\odot} \mathrm{yr}^{-1}\right)$. The important point is that the wind momentum of this star is as low as that of the SMC-N81 stars (see Fig. 16). The metal content of 10 Lac being near solar (10 Lac belongs to the nearby association Lac OB1, and its CNO abundances being between 0.5 and 0.9 the solar values see Villamariz et al. 2002), this indicates that metallicity is not uniquely responsible for the weakness of the winds observed in the present work.

The above discussion shows that our understanding of the metallicity effects on the wind properties of massive stars is still partial and that both observational and theoretical efforts are needed to improve this situation.

\section{3) Decoupling:}

Finally, an alternative answer to the puzzling wind properties of the low luminosity $\mathrm{O}$ stars is that a decoupling between the absorbing ions and the passive plasma may occur. In classical radiatively driven winds, ions from metals gain momentum 
from the photons they absorb and redistribute this momentum to the passive plasma through friction. However, if the density in low enough, friction may become inefficient so that the absorbing ions are the only species to be accelerated. Due to their low abundances compared to $\mathrm{H}$ and $\mathrm{He}$ (constituting most of the passive plasma), the mass loss is greatly reduced.

Various studies have been pursued to determine the conditions under which such decoupling may take place (e.g., Babel 1995, 1996; Krtička \& Kubát 2000). Springmann \& Pauldrach (1992) have estimated that decoupling occurs when radiative acceleration is not compensated by frictional braking. We have applied their Eq. (15) to the case of our SMC-N81 stars and we found that decoupling is expected to happen only in the outer region of the wind where the outflow velocity has almost reached the terminal velocity. In the transsonic region, where the mass loss rate is set, decoupling is not predicted to take place. More recently, Owocki \& Puls (2000) gave an estimate of the wind velocity at which maximal coupling occurs as a function of stellar and wind parameters. If this velocity is greater than the terminal velocity, then the wind can be described by a single component fluid. In the SMC-N81 stars, this maximal drift is predicted to happen at velocities of the order $v_{\infty}$ so that decoupling is not expected to happen. Even more recently, Krtička et al. (2003) have estimated the metallicity below which decoupling should occur due to the reduced wind density for a given set of stellar parameters. In the case of the SMC-N81 stars, this limit is of the order $1 / 100 Z_{\odot}$ which is well below the metallicity of the SMC. As a consequence, it seems that decoupling cannot explain the weakness of the winds.

After these considerations the most likely explanation for the weak winds of the analysed stars is therefore that a breakdown of the modified wind momentum-luminosity relation exists at low luminosity for dwarfs (1), possibly independently of metallicity. A possible mechanism responsible for such a behaviour may exist (cf. above) although no realistic calculations have been done for parameters appropriate to the objects considered here. In any case, it is not clear what parameter(s) would determine that some $\mathrm{O}$ dwarf stars have such weak winds in comparison to other $\mathrm{O}$ stars of similar luminosity. Possible relevant parameters could be a higher gravity, higher mass/light ratio, or others (such as the presence of magnetic fields). From the current limited sample of objects showing such puzzling wind properties we speculate that the low mass loss rate is probably intrinsically related to the youth of the stars. It is conceivable that we are beginning to witness the "onset" of radiatively driven winds in young, still somewhat underluminous $\mathrm{O}$ stars shortly after their formation. Further systematic studies of Vz stars and related objects with indications of weak winds will be necessary to resolve these issues.

\section{Conclusion}

Based on UV spectra obtained with STIS/HST we have analysed the stellar and wind properties of the four main exciting stars of the High Excitation Blob SMC-N81 using extensive calculations of spherically expanding non-LTE line blanketed atmosphere models with the code CMFGEN.
The main results are the following:

$\diamond$ The stellar properties $\left(L, T_{\text {eff }}\right)$ indicate that the SMC-N81 components are young ( 0-4 Myrs old) O stars which show, with perhaps the exception of star 1, a lower luminosity than "normal" Galactic O dwarfs. This, together with the closeness to the ZAMS for star 3 and 11, confirms the conclusion of Paper I that they may belong to the Vz class (Walborn \& Parker 1992).

$\diamond$ The UV spectra of the N81 stars show unusually weak stellar winds. The upper limits on mass loss rates are of the order a few $10^{-9} M_{\odot} \mathrm{yr}^{-1}$ which is low compared to 1) Galactic stars of the same luminosity and 2) the most recent predictions of $\dot{M}$ as a function of stellar parameters and metallicity. Point 1) could be qualitatively understood due to the reduced metallicity of the SMC but point 2) indicates that this reduction is higher than expected.

Although the mass loss rates derived from the UV line analysis are potentially affected by uncertainties in the modeled ionisation fractions, various tests indicate that the above conclusions remain qualitatively valid.

$\diamond$ Our objects show modified wind momenta $\left(M_{\odot} v_{\infty} R^{1 / 2}\right)$ which are, for the same luminosity $L$, lower by typically two orders of magnitude compared to the "normal" O star samples. Similarly low wind momenta have also been found by Bouret et al. (2003) for 3 SMC stars in NGC 346. The modified wind momentum-luminosity relation of all the SMC objects could be interpreted as showing a breakdown at low luminosities or a different slope than the Galactic relation. The current sample of SMC stars may indeed indicate a steeper slope at least for giants and dwarfs, but the scatter is still too large to firmly establish this trend. However, the most recent hydrodynamical models (Vink et al. 2001; Kudritzki 2002; Hoffmann et al. 2002) do not predict such a change in the slope between solar and SMC metallicities. Furthermore we present the first indications that some Galactic objects also have low wind momenta, comparable to the SMC dwarfs. This also tends to exclude explanations based uniquely on metallicity.

$\diamond$ Possible explanations for a breakdown of the modified wind momentum-luminosity relation at low luminosities are discussed. Ionic decoupling appears unlikely according to various estimates. A failure of the CAK parameterisation in high density atmospheres, discussed by Owocki \& Puls (1999), might be invoked to explain a lower acceleration in the transsonic region where the mass loss rate is set. Although the physical mechanism leading to such weak winds remains currently unknown, we speculate that the low mass loss rate is probably intrinsically related to the youth of the stars, possibly testifying to a phase of the "onset" of radiatively driven winds in young $\mathrm{O}$ stars shortly after their formation.

Further studies of very young massive stars, Vz stars, and related objects with indications of weak winds will be of great interest to attempt to understand these puzzling wind properties and to provide interesting constraints on the development of stellar winds in the early phases of massive star evolution or possibly even on the final phases of their birth. 
Acknowledgements. We thank Jean-Claude Bouret, Luc Dessart, Claus Leitherer, André Maeder, Georges Meynet and Stan Owocki for useful discussions. We also thank Artemio Herrero and Gerard Testor who kindly provided respectively the optical spectra of $10 \mathrm{Lac}$ and the integrated optical spectra of SMC-N81 corrected for nebular contamination. Artemio Herrero is also acknowledged for his constructive comments as the referee of the paper. The present results rely heavily on generous allocation of computing time from the CALMIP and IDRIS centers. F.M., D.S., and M.H.-M. thank the French "Programme National de Physique Stellaire" (PNPS) for support. Part of this work was also supported by the French "Centre National de Recherche Scientifique" (CNRS) and by the Swiss National Fund.

\section{References}

Abbott, D. C. 1982, ApJ, 259, 282

Allen, C. W. 1976, Astrophysical Quantities (London: Athlone)

Abbott, D. C., \& Hummer, D. G. 1985, ApJ, 294, 286

Asplund, M. 2003, in CNO in the Universe, ed. C. Charbonnel, D. Schaerer, \& G. Meynet, ASP Conf. Ser., 304, 279

Babel, J. 1995, A\&A, 301, 823

Babel, J. 1996, A\&A, 309, 867

Behrend, R., \& Maeder, A. 2001, A\&A, 373, 190

Bjorkmann, J. E., \& Cassinelli, J. P. 1993, ApJ, 409, 429

Bonnell, I. A., Bate, M. R., \& Zinnecker, H. 1998, MNRAS, 298, 93

Bouret, J. C., Lanz, T., Hillier, D. J., et al. 2003, ApJ, in press [arXiv:astro-ph 0301454]

Chiosi, C., \& Maeder, A. 1986, ARA\&A, 24, 329

Crowther, P. A., Hillier, D. J., Evans, C. J., et al. 2002, ApJ, 579, 774

de Koter, A., Heap, S. R., \& Hubeny, I. 1998, ApJ, 509, 879

di Benedetto, G. P. 1997, ApJ, 486, 60

Drew, J. E. 1985, MNRAS, 217, 867

Dyson, J. E. 1978, A\&A, 62, 269

Fanelli, M. N., O’Connell, R. W., Burstein, D., \& Wu, C. 1992, ApJS, 82,197

Feldmeier, A., Kudritzki, R. P., Palsa, R., Pauldrach, A. W. A., \& Puls, J. 1997, A\&A, 320, 899

Figer, D. F., Najarro, F., Gilmore, D., et al. 2002, ApJ, 581, 258

Fitzpatrick, E. L., \& Savage, B. D. 1983, ApJ, 267, 93

Grevesse, N., \& Sauval, A. 1998, Space Sci. Rev., 85, 161

Heap, S. R. 2003, in CNO in the Universe, ed. C. Charbonnel, D. Schaerer, \& G. Meynet, ASP Conf. Ser., 304, 41

Herrero, A., Puls, J., \& Villamariz, M. R. 2000, A\&A, 354, 193

Herrero, A., Puls, J., \& Najarro, F. 2002, A\&A, 396, 949

Heydari-Malayeri, M., \& Testor, G. 1982, A\&A, 111, L11

Heydari-Malayeri, M., Le Bertre, T., \& Magain, P. 1988, A\&A, 195, 230

Heydari-Malayeri, M., Rosa, M. R., Zinnecker, H., Deharveng, L., \& Charmandis, V. 1999, A\&A, 344, 848

Heydari-Malayeri, M., Rosa, M. R., Schaerer, D., Martins, F., \& Charmandis, V. 2002a, A\&A, 381, 951 (Paper I)

Heydari-Malayeri, M., Charmandis, V., Deharveng, L., et al. 2002b, in A massive star odyssey: from main sequence to supernova, ed. K. A. van der Hucht, A. Herrero, \& C. Esteban, IAU Symp., 212,553

Hill, V. 1999, A\&A, 345, 430

Hillier, D. J., \& Miller, D. L. 1998, ApJ, 496, 407

Hillier, D. J., Lanz, T., Heap, S. R., et al. 2003, ApJ, 588, 1039
Hoffmann, T. L., Pauldrach, A. W. A., \& Puls, J. 2002, in A massive star odyssey: from main sequence to supernova, ed. K. A. van der Hucht, A. Herrero, \& C. Esteban, IAU Symp., 212, 206

Howarth, I. D., \& Prinja, R. K. 1989, ApJS, 69, 527

Hubeny, I., \& Lanz, T. 1995, ApJ, 439, 875

Krtička, J., \& Kubát, J. 2000, A\&A, 359, 983

Krtička, J., Owocki, S. P., Kubát, J., Galloway, R. K., \& Brown, J. C. 2003, A\&A, 402, 713

Kudritzki, R. P., Puls, J., Lennon, D. J., et al. 1999, A\&A, 350, 970

Kudritzki, R. P., \& Puls, J. 2000, ARA\&A, 38, 613

Kudritzki, R. P. 2002, ApJ, 577, 389

Lamers, H. J. G. L. M., Cerruti-Sola, M., \& Perinotto, M. 1987, ApJ, 314,726

Lamers, H. J. G. L. M., Haser, S., de Koter, A., \& Leitherer, C. 1999, ApJ, 516, 872

Lamers, H. J. G. L. M., \& Cassinelli, J. P. 1999, Introduction to stellar winds (Cambridge University Press)

Leitherer, C. 1988, ApJ, 533, 626

Lejeune, T., \& Schaerer, D. 2001, A\&A, 366, 538

Mallouris, C., Welty, D. E., York, D. G., et al. 2001, ApJ, 558, 133

Maeder, A., \& Meynet, G. 2000, ARA\&A, 38, 143

Martins, F., Schaerer, D., \& Heydari-Malayeri, M. 2002a, in A massive star odyssey: from main sequence to supernova, ed. K. A. van der Hucht, A. Herrero, \& C. Esteban, IAU Symp., 212, 564

Martins, F., Schaerer, D., \& Hillier, D. J. 2002b, A\&A, 382, 999

Massey, P., Bresolin, F., Kudritzki, R. P., Puls, J., \& Pauldrach, A. W. A. 2004 , ApJ, in press

Meynet, G., \& Maeder, A. 1997, A\&A, 321, 465

Najarro, F., Krabbe, A., Genzel, R., et al. 1997, A\&A, 325, 700

Norberg, P., \& Maeder, A. 2000, A\&A, 359, 1025

Owocki, S. P., Castor, J. I., \& Rybicki, G. B. 1988, ApJ, 335, 914

Owocki, S. P., \& Puls, J. 1999, ApJ, 510, 355

Owocki, S. P., \& Puls, J. 2000, ApJ, 568, 965

Pauldrach, A. W. A., Kudritzki, R. P., Puls, J., Butler, K., \& Husinger, J. 1994, A\&A, 283, 525

Pauldrach, A. W. A., Hoffmann, T. L., \& Lennon, M. 2001, A\&A, 375 , 161

Prévot, M. L., Lequeux, J., Maurice, E., Prévot, L., \& Rocca-Volmerange, B. 1984, A\&A, 132, 389

Puls, J., Kudritzki, R. P., Herrero, A., et al. 1996, A\&A, 305, 171

Puls, J., Springmann, U., \& Lennon, M. 2000, A\&AS, 141, 23

Repolust, T., Puls, J., \& Herrero, A. 2004, A\&A, 415, 349

Santolaya-Rey, A. E., Puls, J., \& Herrero, A. 1997, A\&A, 323, 488

Schaerer, D., de Koter, A., Schmutz, W., \& Maeder, A. 1996, A\&A, 312,475

Sembach, K. R., \& Savage, B. D. 1992, ApJS, 83, 147

Smith-Neubig, M., \& Bruhweiler, F. 1997, AJ, 114, 1951

Springmann, U. W. E., \& Pauldrach, A. W. A. 1992, A\&A, 262, 515

Vacca, W. D., Garmany, C. D., \& Shull, J. M. 1996, ApJ, 460, 914

Venn, K. A. 1999, ApJ, 518, 405

Vermeij, R., \& van der Hulst, J. M. 2002, A\&A, 391, 1081

Villamariz, M. R., Herrero, A., Becker, S. R., \& Butler, K. 2002, A\&A, 388, 940

Vink, J., de Koter, A., \& Lamers, H. J. G. L. M. 2000, A\&A, 362, 295

Vink, J., de Koter, A., \& Lamers, H. J. G. L. M. 2001, A\&A, 369, 574

Walborn, N., \& Parker, J. 1992, ApJ, 399, L87

Walborn, N., Nichols-Bohlin, J., \& Panek, R. J. 1995, IUE Atlas of O-Type stellar spectra 


\section{Online Material}


F. Martins et al.: Young massive stars in SMC N81, Online Material p 2

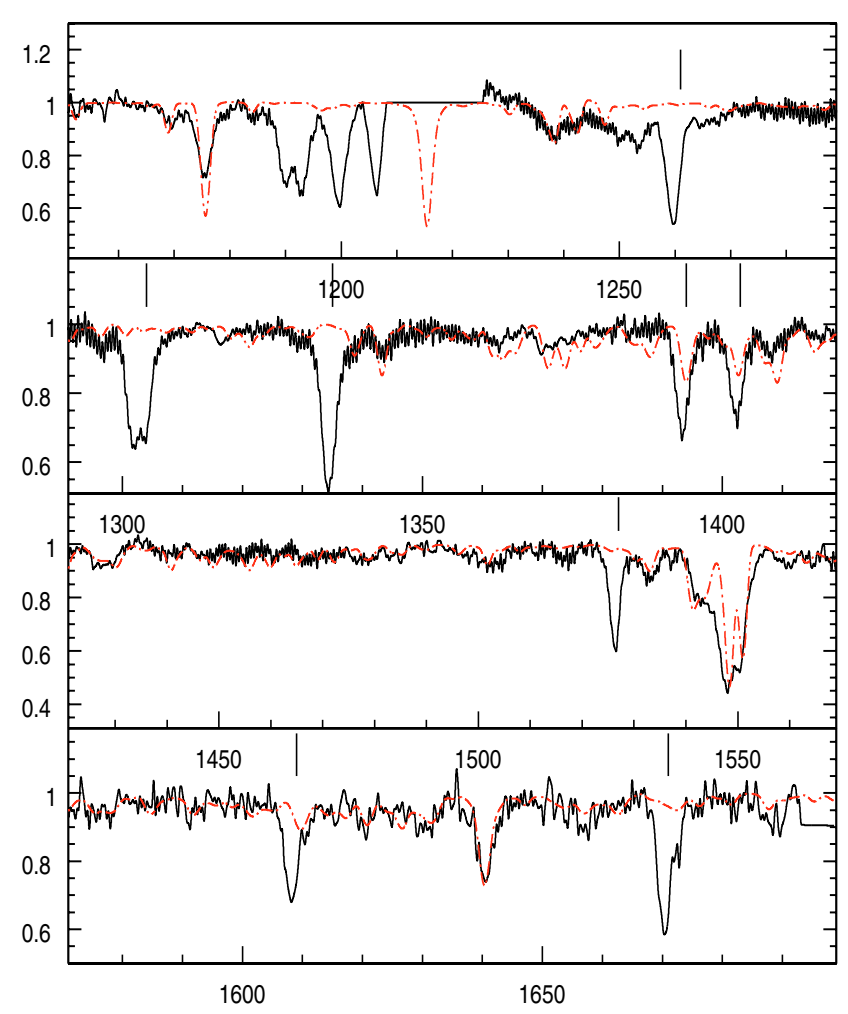

Fig. A.1. Best fit model for star 1. The model parameters are: $T_{\text {eff }}=38500 \mathrm{~K}, \dot{M}=10^{-8.5} M_{\odot} \mathrm{yr}^{-1}, v_{\infty}=1500 \mathrm{~km} \mathrm{~s}^{-1}$, $V \sin i=200 \mathrm{~km} \mathrm{~s}^{-1}, f=0.01$. The abundances are the following: $n(\mathrm{He})=0.1 n(\mathrm{H}), \mathrm{C} / \mathrm{C}_{\odot}=1 / 10, \mathrm{~N} / \mathrm{N}_{\odot}=1 / 20, \mathrm{O} / \mathrm{O}_{\odot}=1 / 5$, and $\mathrm{Si}$, $\mathrm{S}$ and $\mathrm{Fe}$ abundances are $1 / 8$ the solar values. Solid line: observations; dot-dashed line: model. The vertical lines indicate the main interstellar lines.

\section{Appendix A: Best fits}

Figures A.1- A.4 give the best fits achieved for star 1, 2, 11 and 3 respectively. For each figure, the model parameters are given and the main interstellar lines are indicated. The interstellar CIV absorption has been added to the synthetic spectra as described in Sect. 4. Even with this correction, the fit of the C IV $\lambda \lambda 1548,1551$ line remains poor for star 2 , showing the difficulty to produce significant absorption without emission. The normalisation of the observed spectra below $1200 \AA$ is very uncertain so that any comparison with models in this wavelength range is irrelevant.

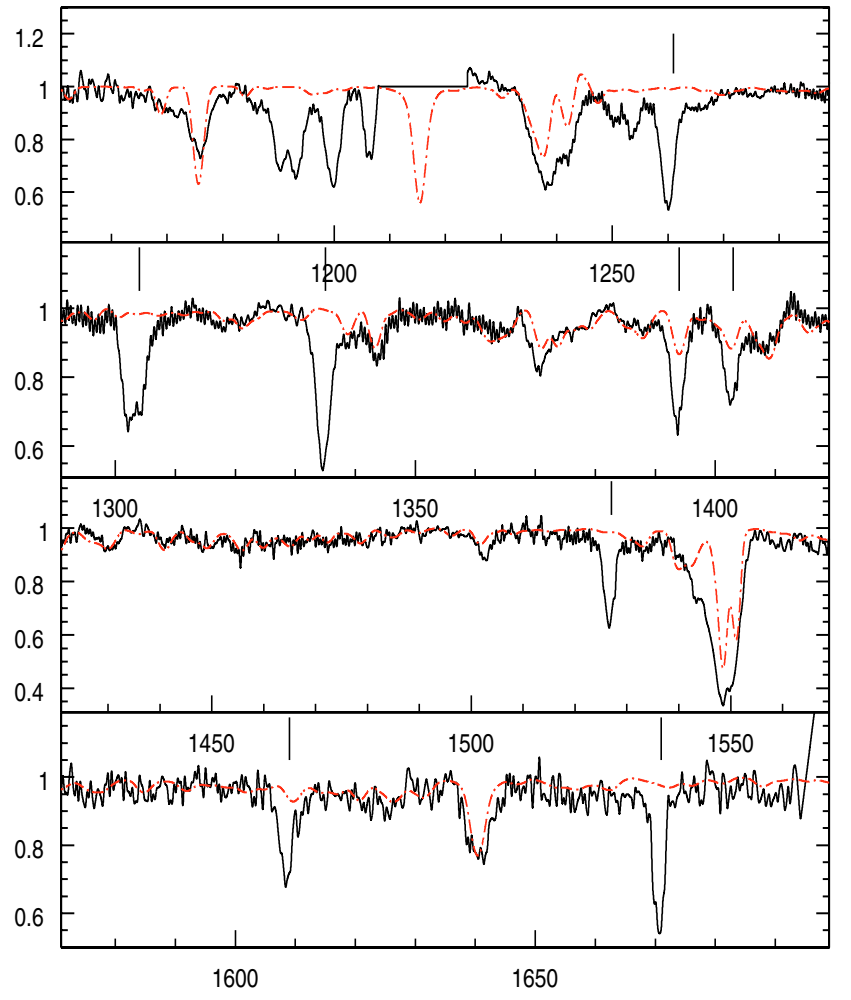

Fig. A.2. Best fit model for star 2. The model parameters are: $T_{\text {eff }}=40000 \mathrm{~K}, \dot{M}=10^{-8.5} M_{\odot} \mathrm{yr}^{-1}, v_{\infty}=1800 \mathrm{~km} \mathrm{~s}^{-1}$, $V \sin i=300 \mathrm{~km} \mathrm{~s}^{-1}, f=0.01$. The abundances are the following: $n(\mathrm{He})=0.1 n(\mathrm{H})$, and $\mathrm{C}, \mathrm{N}, \mathrm{O}, \mathrm{Si}, \mathrm{S}$ and $\mathrm{Fe}$ abundances are $1 / 8$ the solar values. Solid line: observations; dot-dashed line: model. The vertical lines indicate the main interstellar lines. The poor fits of the $\mathrm{C}$ IV $\lambda \lambda 1548,1551$ and $\mathrm{N} v \lambda 1238,1242$ lines comes from the difficulty to have important absorption without emission. 
F. Martins et al.: Young massive stars in SMC N81, Online Material p 3

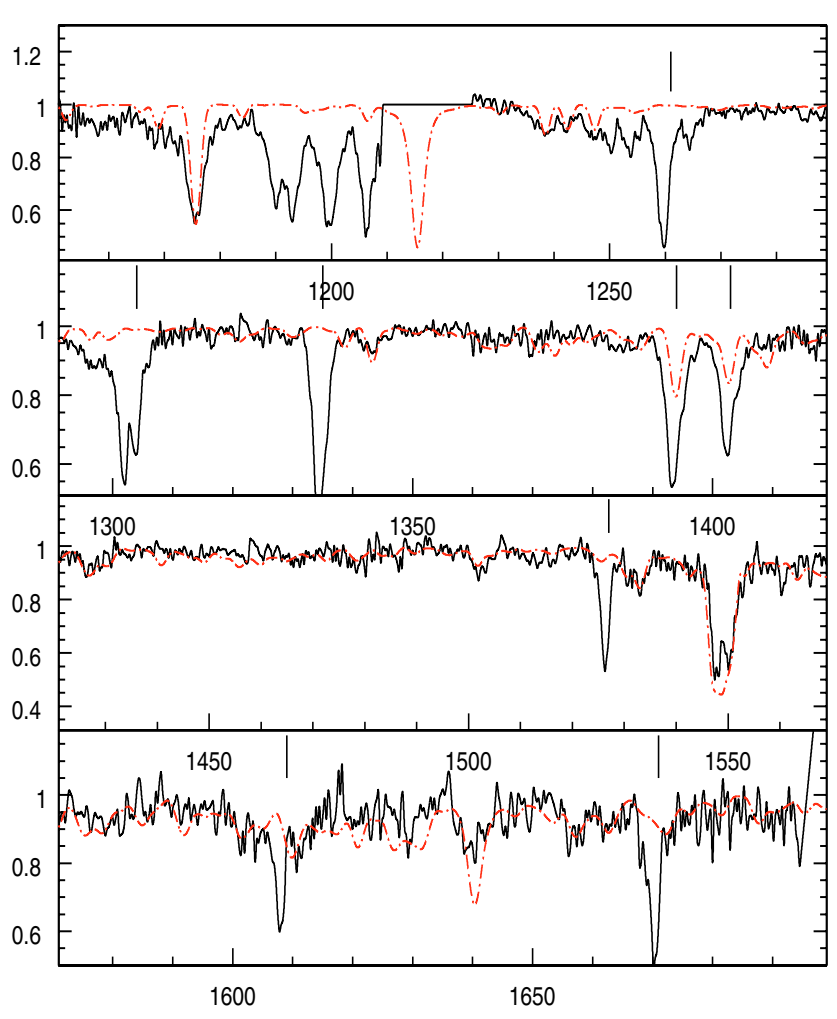

Fig. A.3. Best fit model for star 3. The model parameters are: $T_{\text {eff }}=36000 \mathrm{~K}, \dot{M}=10^{-8.5} M_{\odot} \mathrm{yr}^{-1}, v_{\infty}=300 \mathrm{~km} \mathrm{~s}^{-1}$, $V \sin i=250 \mathrm{~km} \mathrm{~s}^{-1}$. The abundances are the following: $n(\mathrm{He})=$ $0.1 n(\mathrm{H}), \mathrm{C} / \mathrm{C}_{\odot}=1 / 10, \mathrm{~N} / \mathrm{N}_{\odot}=1 / 20, \mathrm{O} / \mathrm{O}_{\odot}=1 / 5$, and $\mathrm{Si}, \mathrm{S}$ and $\mathrm{Fe}$ abundances are 1/8 the solar values. Solid line: observations; dot-dashed line: model. The vertical lines indicate the main interstellar lines.

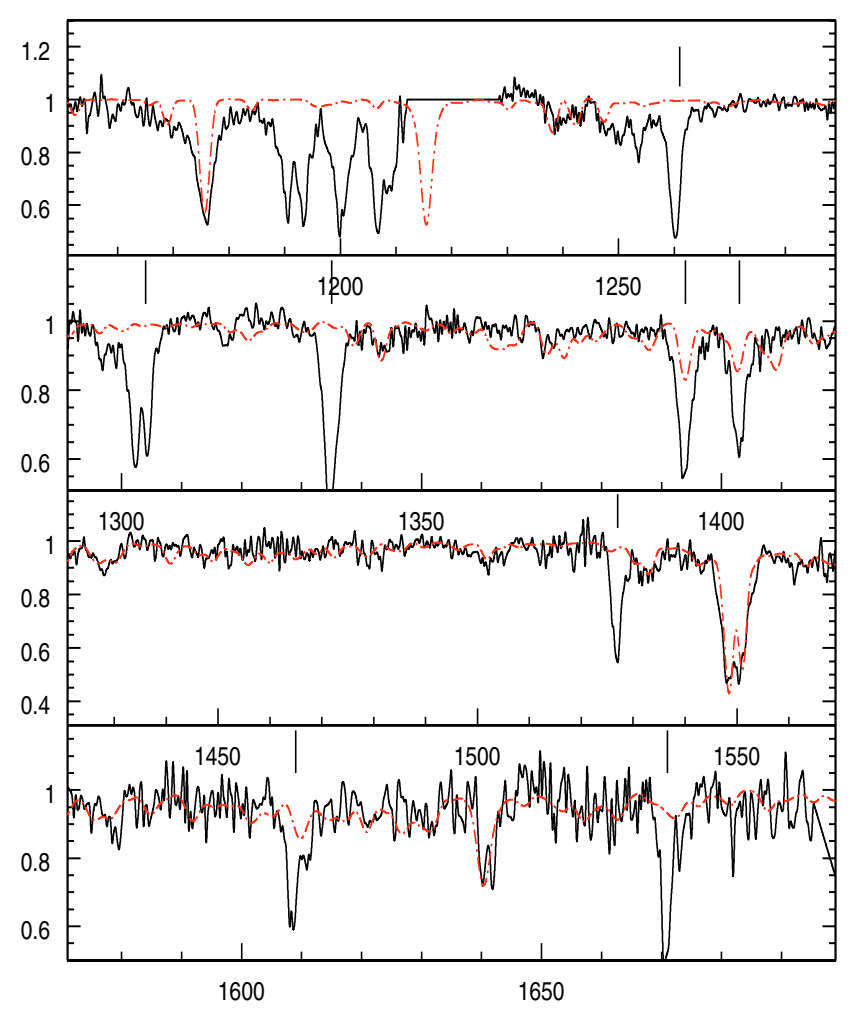

Fig. A.4. Best fit model for star 11. The model parameters are: $T_{\text {eff }}=37000 \mathrm{~K}, \dot{M}=10^{-9.5} M_{\odot} \mathrm{yr}^{-1}, v_{\infty}=600 \mathrm{~km} \mathrm{~s}^{-1}$, $V \sin i=250 \mathrm{~km} \mathrm{~s}^{-1}$. The abundances are the following: $n(\mathrm{He})=$ $0.1 n(\mathrm{H}), \mathrm{C} / \mathrm{C}_{\odot}=1 / 10, \mathrm{~N} / \mathrm{N}_{\odot}=1 / 20, \mathrm{O} / \mathrm{O}_{\odot}=1 / 5$, and $\mathrm{Si}, \mathrm{S}$ and $\mathrm{Fe}$ abundances are 1/8 the solar values. Solid line: observations; dot-dashed line: model. The vertical lines indicate the main interstellar lines. 